\title{
The anisotropy of hexagonal closed-pack and liquid interface free energy using molecular dynamics simulations based on modified embedded-atom method
}

\author{
Ebrahim Asadi ${ }^{\mathrm{a}^{*}}$ and Mohsen Asle Zaeem ${ }^{\mathrm{b} \dagger}$ \\ a Department of Mechanical Engineering, The University of Memphis, Memphis, TN 38152, USA \\ ${ }^{\mathrm{b}}$ Department of Materials Science and Engineering, Missouri University of Science and Technology, \\ Rolla, MO 65409, USA
}

\begin{abstract}
This work aims to comprehensively studythe anisotropy of the hexagonal close-packed (HCP)-liquid interface free energy using molecular dynamics (MD) simulations based on the modified-embedded atom method (MEAM).As a case study, all the simulations are performed for Magnesium (Mg). The solid-liquid coexisting approach is used to accurately calculate the melting point and melting properties. Then, the capillary fluctuation method (CFM) is used to determine theHCP-liquid interface free energy $(\gamma)$ and anisotropy parameters. In CFM, a continuous order parameter isemployed to accurately locate the HCP-liquid interface location, andthe HCPsymmetry-adapted spherical harmonics areused to expand $\gamma$ in terms of its anisotropy parameters $\left(\varepsilon_{20}, \varepsilon_{40}, \varepsilon_{60}\right.$ and $\left.\varepsilon_{66}\right)$.Eight slip and twinning planes (basal, two prismatic, two pyramidal, and three twinning planes) areconsidered as the HCP-liquid interface planes. An average HCP-liquid interface free energy of $122.2\left(\mathrm{~mJ} / \mathrm{m}^{2}\right)$, non-zero $\varepsilon_{20}, \varepsilon_{40}$, and $\varepsilon_{66}$ parameters, and approximately zero $\varepsilon_{60}$ parameter for $\mathrm{Mg}$ are predicted. Using thesefindings, the first preferred dendrite growth direction in solidification of $\mathrm{Mg}$ is predicted as [1120], which is in agreement with experiments. Also, a second preferred dendrite growth direction for Mgis predicted as $[33 \overline{6} 2]$.
\end{abstract}

Keywords:Interface free energy; Spherical harmonics; MEAM;Hexagonal close-packed; Magnesium.

\footnotetext{
*E-mail address: easadi@memphis.edu(E. Asadi), Tel.: +1 901678 3332; fax: +1 9016785459.

†E-mail address: zaeem@mst.edu(M. Asle Zaeem),Tel.: +1 573341 7184; fax: +1 5733416934.
} 


\section{Introduction}

Over the years, different computational models at different length and time scales have been usedto study the solid-liquid coexistence of metals to obtain better understanding of this phenomenon as well as the formation and evolution of the subsequent microstructures and defect patterns[1]. Among these models, molecular dynamics (MD) is of a special interest because of its reasonable computational cost and its capability in accurate calculations of the solid-liquid interface properties[2]. The accurate calculation of the solid-liquid properties, such as interface free energy $(\gamma)$ and its anisotropy, is of crucial importance, because these properties are the key factors influencing the solid phase selection and crystal nucleation from the melt.Since this article aims to study theHCP-liquid coexistence, we will mostly focus our discussion on this class of materials and MD simulations only.

The first work that used MD simulations to study the solid-liquid interface properties is the pioneering work of Broughton and Gilmer[3]. They used Lennard-Jones FCC-liquid systems at the triple point for their MD simulations and calculated $\gamma$ to be the aggregated work required to cleave and combine solid and liquid systems. Their technique later refined by Davidchack and Laird $[4,5]$ and it is called the cleaving technique. However, the major MD effort dealing with the HCP-liquid coexistence simulations has been based on the capillary fluctuation method (CFM)[6]. CFM is based on equilibrating a coexisting solid-liquid structure at the exact melting point of the material, determining the location of the solid-liquid interface, and finding the Fourier modes of the solid-liquid interface and using them to determine the solid-liquid interface stiffness $\left(\gamma+\gamma^{\prime \prime}\right)[2,7-9]$. Then, the anisotropy of the system is explained by expanding $\gamma$ in terms of spherical harmonics for the considered crystal symmetry. Sun et al. [6] extended CFM for HCP metals by considering only a basal and two prismatic planes as the HCP-liquid interface planes in their simulationstodescribethe anisotropy of $\gamma$. They used $\mathrm{Mg}$ as their case study and embedded-atom method (EAM) [10]as the interatomic potential intheir MD simulations; EAM is a semi-empirical many-body potential for the atomistic simulations of metallic systems.Until now and to the knowledge of the authors, the work of Sun and coworkers is the only MD work to determine $\gamma$ and its anisotropy for HCP metals. The other works related to the MD simulations of HCP-liquid coexisting systems include the study of the melting[11-13], interface properties [14] and kinetic mobility $[15,16]$. 
The first objective of this article is to provide another perspective to the MD calculations of $\gamma$ and its anisotropy for HCP metals by usinga different class of interatomic potentials,MEAM potentials[17], in MD simulations.MEAMadds the directionality of bonding in covalent materials to the EAM formalism. Today, the MEAM potential is widely used in the computational materials science and engineering community to simulate unary, binary, ternary, and multicomponent metallic systems[18]. Since only EAM-MD simulations have been used to determine the HCP-liquid interface properties, using MEAM-MD simulations in this study provides another perspective and more confidence in using MD simulations for this purpose. In fact,we recently used MEAM to study the solid-liquid coexistence of body-centered cubic (BCC) and facecentered cubic (FCC) metals [19, 20], and we showed that the calculated melting properties, solid-liquid interface free energy, and surface anisotropyare in a very good agreement with the experimental data.Another objective of this work is to more comprehensively study the HCPliquid coexistence by including all of the deformation planes in HCP crystals as the HCP-liquid interface planes(eight different slip and twining planes); this wasmissing in the previousstudies.

In this work, we use one set of MEAM parameters for $\mathrm{Mg}$ in all of the MD simulations[21].These MEAM parameters were tested before showing reasonable predictions of a variety of low and high temperature properties such as elastic constants, structural energy differences, vacancy formation energy, stacking fault energies, surface energies, melting point, specific heat, and thermal expansion coefficient.First, the solid-liquid coexistence approach is used to accurately calculate the melting point which is crucial for the rest of the simulations. Second, the relations for $\gamma$ and $\gamma+\gamma^{\prime \prime}$ in the concept of CFM for twelve slabs with different crystallographic orientations arederived by expanding the anisotropy of $\gamma$ in terms of HCPsymmetry-adaptedspherical harmonics. Third, a continuous order parameter is used to locate the interface location in MD simulations of HCP-liquid coexisting slabs, andthe interface stiffnessis calculated for each case. Fourth, statistical average HCP-liquid interface free energy and anisotropy parameters for $\mathrm{Mg}$ arecalculated using the results of $\mathrm{MD}$ simulations and HCPsymmetry-adaptedspherical harmonics. And finally,a discussion on the preferred dendrite growth direction and rate during the solidification of $\mathrm{Mg}$ are provided as the results of the predictions madein this study. 


\section{Melting properties}

\subsection{Interatomic potential}

The second nearest-neighbor $(2 \mathrm{NN})$ formalism of MEAM [22, 23]is used as the interatomic potential for all the MD simulations in this study. In the MEAM formalism, the total energy of a single-element system is given by

$$
E_{t o t}=\sum_{i}\left[F_{i}\left(\bar{\rho}_{i}\right)+\frac{1}{2} \sum_{j(\neq i)} S_{i j} \phi_{i j}\left(R_{i j}\right)\right]
$$

where $F_{i}$ is the "embedding energy" function (energy required to embed an atom in the background electron density $\bar{\rho}_{i}$ at site $\left.i\right), S_{i j}$ is the screening factor between atoms at sites $i$ and $j$, and $\phi_{i j}$ is the pair interaction between atoms at sites $i$ and $j$ with a separation distance of $R_{i j}$. The details of the MEAM formalism have been presented in other publications devoted to the development of the potential[24]. MEAM for a single element system has fourteen parameters in its formalism which needto be determined for theselected material. We use the 2NN-MEAM parameters for Mg which were presented previously by Kim et al. [21];theyshowed that using these parametersresults in reasonable calculations of the low and high temperature properties of $\mathrm{Mg}$, which are comparable to the experimental andab initioMD data. The properties considered for the development of these MEAM parameters for $\mathrm{Mg}$ were: cohesive energy, lattice parameters, elastic constants, structural energy differences, vacancy formation and migration energies, divacancy formation energy, self-interstitial energy, stacking fault energies, surface energies, specific heat, thermal expansion, melting point, latent heat, and expansion in melting. Since the focus of this study is to use MEAM for the two-phase solid-liquid coexistence simulations, it is critical to accurately determine the melting point for the potential used in the MD simulations. Therefore, we use the solid-liquid coexisting approach to accurately recalculate the melting properties. Hence, we expect a small difference in our calculations of the melting point, latent heat, and expansion in melting compared with those calculated by Kim et al. [21].

In our simulations, the large-scale atomic/molecular massively parallel simulator (LAMMPS) MD code [25] is used. Periodic boundary conditions anda time step size of 0.002 psare used for all the MD simulations.

\subsection{Melting properties}


Eight different planes (Fig. 1) are chosen as the HCP-liquid interface in this work. The labeling shown in Fig. 1 will be used throughout this work to refer to different crystallographic configurations. The configurations shown in Fig. 1have been identified as the active deformation planes for Mg (slip and twinning systems)[26]. To the date, only Basal, Prismatic I and II planes (Fig. 1a-c, respectively) have been used to study the HCP-liquid interface properties[6].The method for accurately calculating the melting point of BCC and FCC metals using MD simulations has been extensively explained in the literature (e.g., Ref.[27]). The method is typically based on constructing an equilibrating two-phase solid-liquid simulation box; however, the procedure needs slight modifications for HCP metals. First,a simulation box consisting of $m \times n \times l$ periodic solid cells is equilibrated at an estimated melting point of the material using previously-calculated lattice parameters (both $a$ and $c / a$ ). Canonical ensemble (NVT) with a Nòse-Hoover thermostat[28, 29] is used for this step, and $l$ direction is normal to the HCP-liquid interface and its length is roughly ten times longer thanthe other two dimensions. The cell sizes $m$ and $n$ are chosen to form a nearly square area for the HCP-liquid interface. Then, the central half of the simulation box is melted at a high temperature by running a dynamics simulation using NVT ensemble, while holding the other half fixed. The melted half of the simulation box is equilibrated at the estimated melting point using an isothermal-isobaric (NPT) ensemble for 0.5 ns, while the box size at the normal direction is allowed to relax only for the melted half. To minimize the pressure in all directions, the entire simulation box is then allowed to relax in the normal direction for $1 \mathrm{~ns}$ using an NPT ensemble at the estimated melting point. Finally, the refined value of the melting point is calculated using an isenthalpic ensemble (NPH) simulation lasting for a considerable amount of time ( $10 \mathrm{~ns})$, while the size of the box in the normal direction is allowed to relax (to minimize the normal pressure). The whole process is repeated using the calculated refined temperature until convergence is achieved.The calculated melting pointsutilizing the explained methodarepresentedin Table 1. For the case that the interface plane is the Basal plane, the simulations are repeated for two different box sizes. The calculated melting points are $937.25 \mathrm{~K}$ for the simulation box with 3880 atoms and 937.44 for the simulation box with 92160 atoms. The difference between calculations is $\sim 0.02 \%$, which is negligible in the context of MD simulations; thus, the melting point calculations are size independent. We have repeated a similar study to calculatethe melting point for other orientations, listed inTable (1), to ensure the size-independency of the reported results. The 
simulation box for all the eight cases with different interface planes consists of about 100,000 atoms. The required number of atoms in the simulation box for size-independency of the calculated results is also consistent with those used in similar studies [20,30]. Table 1 shows that the variation of the calculated melting point with respect to the interface plane is negligible; thus, the melting point calculations are also orientation independent. The melting point of $\mathrm{Mg}$ is determined to be the average of the calculated melting points reported in Table 1, which is 937.9 K.

Table 1. Calculated melting point for different choices of the HCP-liquid interface plane.

\begin{tabular}{lccc}
\hline \hline Interface Plane & Box size $(\mathrm{nm})$ & Number of Atoms & $T_{M}(\mathrm{~K})$ \\
\hline Basal & $3.96 \times 5.14 \times 48.10$ & 38880 & 937.25 \\
Basal & $5.28 \times 6.85 \times 64.13$ & 92160 & 937.44 \\
\hline Prismatic I & $5.28 \times 6.41 \times 68.53$ & 92160 & 938.07 \\
Prismatic II & $6.85 \times 6.41 \times 52.76$ & 92160 & 938.12 \\
Pyramidal I & $5.28 \times 7.27 \times 67.99$ & 103680 & 937.90 \\
Pyramidal II & $6.85 \times 6.91 \times 56.95$ & 107184 & 937.46 \\
Twinning I & $5.28 \times 6.26 \times 76.06$ & 99840 & 937.80 \\
Twinning II & $6.85 \times 6.71 \times 65.21$ & 119232 & 938.51 \\
Twinning III & $5.28 \times 8.08 \times 67.97$ & 115200 & 938.08 \\
\hline
\end{tabular}

Themelting point $\left(T_{M}\right)$, latent heat $(L)$, liquid density at the melting point $\left(\rho_{l}\right)$, and expansion in melting ( $\Delta V_{\text {melting }}$ ) for Mg calculated by MEAM and two EAM potentials (labeled as LEAM and EAM),along with experimental counterparts are listed in Table 2. MEAM and EAM both calculate the melting point, latent heat, and expansion in melting in agreement with the experiments. However, EAM prediction of the liquid density contains $151.3 \%$ error, while MEAM also predicts the liquid density in good agreement with experiment $(5.1 \%$ error). It is worth mentioning that, MEAM-MD simulations calculate $L, \rho_{l}$, and $\Delta V_{\text {melting }}$ at the experimental melting-point $(923.2 \mathrm{~K})$ to be $10.1 \mathrm{~kJ} / \mathrm{mol}, 0.037$ atom $/ \AA^{3}$, and $1.80 \AA^{3} /$ atom, respectively.

Table 2.Melting-pointproperties of Mgcalculated by MEAM-MD compared with the results of other MD methods and experiments. The values in the parentheses are the errors comparing with the experimental data. 


\begin{tabular}{lllll}
\hline \hline Method & $T_{M}(\mathrm{~K})$ & $\mathrm{L}(\mathrm{kJ} / \mathrm{mol})$ & $\rho_{l}\left(\right.$ atom $\left./ \AA^{3}\right)$ & $\Delta V\left(\AA^{3} /\right.$ atom $)$ \\
\hline MEAM;present & $937.9(1.6 \%)$ & $10.2(13.3 \%)$ & $0.037(5.1 \%)$ & $1.84(64.3 \%)$ \\
LEAM; [6] & $745(19.3 \%)$ & $7.0(22.2 \%)$ & $0.073(87.2 \%)$ & $1.23(9.8 \%)$ \\
EAM; [6] & $914(1.0 \%)$ & $9.5(5.6 \%)$ & $0.098(151.3 \%)$ & $0.65(42.0 \%)$ \\
Exp.; [26] & 923.2 & $8.8-9.2$ & 0.039 & 1.12 \\
\hline
\end{tabular}

\section{HCP-liquid interface free energy}

We utilize CFM [2] to determine $\gamma$ and its anisotropy for Mg. The first step in using CFM is to construct two-phase solid-liquid coexisting slabs which is schematically shown in Fig. 2.The local normal to the solid-liquid interface $(\hat{n})$ forms an angle, $\alpha$, with its average orientation. CFM is based on the calculations of the interface stiffness $\left(\gamma+\gamma^{\prime \prime}\right)$ by two methods,where $\gamma^{\prime \prime}$ is the second derivative of $\gamma$ with respect to $\alpha$. The first method uses the symmetry-adapted spherical harmonics to expand $\gamma$ in terms of its anisotropy parameters and determine the relations for the interface stiffness. The second methodcalculatesthe interface stiffness using MD simulations. Eventually, $\gamma$ and its anisotropy parameters are calculated by comparing the interface stiffness determined from the explained methods. CFM results in an accurate determination of the anisotropy of $\gamma$, because interface stiffness is typically an order of magnitude more anisotropic than the interface free energy.

\subsection{Spherical harmonics}

An arbitrary square integrable function on unit sphere can be expanded in terms of spherical harmonics as[31]

$$
f(\theta, \phi)=\sum_{i=0}^{\infty} \sum_{j=-i}^{i} f_{i j} y_{i j}(\theta, \phi),
$$

where $f_{i j}$ are the expansion coefficients, $y_{i j}(\theta, \phi)$ are the spherical harmonic functions of order $i$ and $j, \theta \in[0, \pi]$ is the colatitudinal coordinate, and $\phi \in[0,2 \pi]$ is the longitudinal coordinate. The crystal symmetry implies that $f_{i j}$ coefficients are non-zero for only a selection of $i$ and $j$. HCP crystal belongs to the $6 / \mathrm{mmm}\left(\mathrm{D}_{6 \mathrm{~h}}\right)$ point group symmetry; thus, $i=6 m$ and $j=2 n$, where $m$ and $n$ are integers [32].Consequently, the HCP-liquid interface free energy can be expanded by considering the HCP crystal symmetry and the double-series expansion in Eq. (2) as 


$$
\gamma(\theta, \phi)=\gamma_{0}\left[1+\varepsilon_{20} \mathrm{y}_{20}(\theta, \phi)+\varepsilon_{40} \mathrm{y}_{40}(\theta, \phi)+\varepsilon_{60} \mathrm{y}_{60}(\theta, \phi)+\varepsilon_{66} \mathrm{y}_{66}(\theta, \phi)\right],
$$

where the expansion includes spherical harmonic functions up to $i=6$ which has been shown by Sun et al. [6] to be sufficient to describe the anisotropy of the HCP-liquid interface free energy. In Eq. (3), $\varepsilon_{20}, \varepsilon_{40}, \varepsilon_{60}$, and $\varepsilon_{66}$ are anisotropy parameters, $\gamma_{0}$ is the average interface free energy, and

$$
\begin{aligned}
& y_{20}=\frac{1}{4} \sqrt{\frac{5}{\pi}}\left[3 \cos (\theta)^{2}-1\right], \\
& y_{40}=\frac{3}{16} \sqrt{\frac{1}{\pi}}\left[35 \cos (\theta)^{4}-30 \cos (\theta)^{2}+3\right], \\
& y_{60}=\frac{1}{32} \sqrt{\frac{13}{\pi}}\left[231 \cos (\theta)^{6}-315 \cos (\theta)^{4}+105 \cos (\theta)^{2}-5\right], \\
& y_{66}=\frac{1}{64} \sqrt{\frac{6006}{\pi}} \sin (\theta)^{6} \cos (6 \phi) .
\end{aligned}
$$

The four HCP symmetry-adaptedspherical harmonics $\left(y_{20}, y_{40}, y_{60}\right.$, and $\left.y_{66}\right)$ are plotted in Fig. 3. We use twelve different slabs for the rest of the study,where the slabs orientations are listed and labeled in Table 3. For instance, $(0001)[11 \overline{2} 0]$ orientation means that the interface plane is $(0001)$ and the width of the slab is parallel to the $[11 \overline{2} 0]$ direction. The third and fourth columns of Table 3 showthe relations forfree energy and stiffness of HCP-liquid interfaces for each orientation in terms of the anisotropy parameters. It is worthy to note that the angles $\theta$ and $\phi$ for each orientation are measured such that the normal to the HCP-liquid interface coincides with the $c$ axis and the basal plane becomes the HCP-liquid interface. Sun et al. [6] presented the relations for the interface stiffness of the first four orientations (Basal, Prismatic I-a and b, Prismatic II-a and b) listed in Table 3, which are in agreement with our derivations.

Table 3. Relations for free energy $(\gamma)$ and stiffness $\left(\gamma+\gamma^{\prime \prime}\right)$ of HCP-liquid interfaces obtained from the expansion of $\gamma$ by HCP symmetry-adopted spherical harmonics. 


\begin{tabular}{|c|c|c|c|}
\hline Basal & $(0001)[11 \overline{2} 0]$ & $\frac{1}{2} \bar{\varepsilon}_{20}{ }^{*}+\frac{3}{2} \bar{\varepsilon}_{40}{ }^{*}+\frac{1}{2} \bar{\varepsilon}_{60}{ }^{*}$ & $-\bar{\varepsilon}_{20}-\frac{27}{2} \bar{\varepsilon}_{40}-10 \bar{\varepsilon}_{60}$ \\
\hline Prismatic I-a & $(1 \overline{1} 00)[11 \overline{2} 0]$ & $-\frac{1}{4} \bar{\varepsilon}_{20}+\frac{9}{16} \bar{\varepsilon}_{40}-\frac{5}{32} \bar{\varepsilon}_{60}+\frac{1}{64} \bar{\varepsilon}_{66}{ }^{*}$ & $-\frac{1}{4} \bar{\varepsilon}_{20}+\frac{9}{16} \bar{\varepsilon}_{40}-\frac{5}{32} \bar{\varepsilon}_{60}-\frac{35}{64} \bar{\varepsilon}_{66}$ \\
\hline Prismatic I-b & $(1 \overline{1} 00)[0001]$ & $-\frac{1}{4} \bar{\varepsilon}_{20}+\frac{9}{16} \bar{\varepsilon}_{40}-\frac{5}{32} \bar{\varepsilon}_{60}-\frac{1}{64} \bar{\varepsilon}_{66}$ & $\frac{5}{4} \bar{\varepsilon}_{20}-\frac{171}{16} \bar{\varepsilon}_{40}+\frac{205}{32} \bar{\varepsilon}_{60}+\frac{5}{64} \bar{\varepsilon}_{66}$ \\
\hline Prismatic II-a & $(11 \overline{2} 0)[\overline{1} 100]$ & $-\frac{1}{4} \bar{\varepsilon}_{20}+\frac{9}{16} \bar{\varepsilon}_{40}-\frac{5}{32} \bar{\varepsilon}_{60}-\frac{1}{64} \bar{\varepsilon}_{66}$ & $-\frac{1}{4} \bar{\varepsilon}_{20}+\frac{9}{16} \bar{\varepsilon}_{40}-\frac{5}{32} \bar{\varepsilon}_{60}+\frac{35}{64} \bar{\varepsilon}_{66}$ \\
\hline Pris & $(11 \overline{2}$ & $-\frac{1}{4} \bar{\varepsilon}_{20}+\frac{9}{16} \bar{\varepsilon}_{40}-\frac{5}{32} \bar{\varepsilon}_{60}+\frac{1}{64} \bar{\varepsilon}_{66}$ & $\frac{5}{4} \bar{\varepsilon}_{20}-\frac{171}{16} \bar{\varepsilon}_{40}+\frac{205}{32} \bar{\varepsilon}_{60}-\frac{5}{64} \bar{\varepsilon}_{66}$ \\
\hline Pyramidal I-a & $(1 \overline{1} 01)[11 \overline{2} 0]$ & $-0.084 \bar{\varepsilon}_{20}-0.363 \bar{\varepsilon}_{40}+0.166 \bar{\varepsilon}_{60}-0.007 \bar{\varepsilon}_{66}$ & $-0.084 \bar{\varepsilon}_{20}-0.363 \bar{\varepsilon}_{40}+0.166 \bar{\varepsilon}_{60}-0.258 \bar{\varepsilon}_{66}$ \\
\hline Pyramidal I-b & $(1 \overline{1} 01)[\overline{1} 102]$ & $-0.084 \bar{\varepsilon}_{20}-0.363 \bar{\varepsilon}_{40}+0.166 \bar{\varepsilon}_{60}+0.007 \bar{\varepsilon}_{66}$ & $0.750 \bar{\varepsilon}_{20}+5.693 \bar{\varepsilon}_{40}-6.817 \bar{\varepsilon}_{60}+0.026 \bar{\varepsilon}_{66}$ \\
\hline Pyramidal II-a & $(11 \overline{2} 2)[\overline{1} 100]$ & $-0.043 \bar{\varepsilon}_{20}-0.490 \bar{\varepsilon}_{40}+0.152 \bar{\varepsilon}_{60}-0.006 \bar{\varepsilon}_{66}$ & $-0.043 \bar{\varepsilon}_{20}-0.490 \bar{\varepsilon}_{40}+0.152 \bar{\varepsilon}_{60}+0.208 \bar{\varepsilon}_{66}$ \\
\hline Pyramidal II-b & $(11 \overline{2} 2)[\overline{1} \overline{1} 21]$ & $-0.043 \bar{\varepsilon}_{20}-0.490 \bar{\varepsilon}_{40}+0.152 \bar{\varepsilon}_{60}-0.002 \bar{\varepsilon}_{66}$ & $0.630 \bar{\varepsilon}_{20}+8.193 \bar{\varepsilon}_{40}-6.485 \bar{\varepsilon}_{60}+0.036 \bar{\varepsilon}_{66}$ \\
\hline Twinning I & $(1 \overline{1} 02)[\overline{1} 101]$ & $0.150 \bar{\varepsilon}_{20}-0.571 \bar{\varepsilon}_{40}-0.111 \bar{\varepsilon}_{60}-0.002 \bar{\varepsilon}_{66}$ & $0.051 \bar{\varepsilon}_{20}+12.315 \bar{\varepsilon}_{40}+3.417 \bar{\varepsilon}_{60}-0.044 \bar{\varepsilon}_{66}$ \\
\hline Twinning II & $(11 \overline{2} 0)[\overline{1} \overline{1} 22]$ & $-0.185 \bar{\varepsilon}_{20}+0.123 \bar{\varepsilon}_{40}+0.059 \bar{\varepsilon}_{60}+0.012 \bar{\varepsilon}_{66}$ & $1.055 \bar{\varepsilon}_{20}-3.122 \bar{\varepsilon}_{40}-2.128 \bar{\varepsilon}_{60}-0.026 \bar{\varepsilon}_{66}$ \\
\hline Twinning III & $(1 \overline{1} 03)[11 \overline{2} 0]$ & $0.290 \bar{\varepsilon}_{20}-0.086 \bar{\varepsilon}_{40}-0.202 \bar{\varepsilon}_{60}+0.0003 \bar{\varepsilon}_{66}$ & $-0.37 \bar{\varepsilon}_{20}+7.141 \bar{\varepsilon}_{40}+8.776 \bar{\varepsilon}_{60}+0.025 \bar{\varepsilon}_{66}$ \\
\hline
\end{tabular}

$$
{ }^{*} \bar{\varepsilon}_{20}=\sqrt{\frac{5}{\pi}} \varepsilon_{20}, \quad \bar{\varepsilon}_{40}=\sqrt{\frac{1}{\pi}} \varepsilon_{40}, \quad \bar{\varepsilon}_{60} \sqrt{\frac{13}{\pi}} \varepsilon_{60}, \quad \bar{\varepsilon}_{66}=\sqrt{\frac{6006}{\pi}} \varepsilon_{66}
$$

\subsection{Interface stiffness}

Calculating the interface stiffness using MD simulations consists of two major steps: a) determining the interface height of the two-phase HCP-liquid coexisting slabs (Fig. 1), where the thickness of the slab is small comparing to its width ( $b \square W$ ), and b) calculating the Fourier amplitudes of the interface height, $A(k)$, and relating it to the interface stiffness using the following well-known relation

$$
\gamma+\gamma^{\prime \prime}=\frac{k_{B} T_{M}}{b W\left\langle|A(k)|^{2}\right\rangle k^{2}} .
$$

In Eq. (5), $k_{B}$ is the Boltzmann constant, $k$ is the Fourier wave-length, andthe time-averaged Fourier amplitudes are used in the relation because of the inherent fluctuations associated with all the MD simulations, especially those simulations performed at high temperatures. It must be noted here that Eq. (5) is only valid for long wave-length limit (or when $1 / k^{2}$ is small) $[30,33$, 34], because the relation between $1 / k^{2}$ and interface stiffness for small-wave-length limitbecomes logarithmic rather than linear. Such a logarithmic finite-size effect has been welldemonstrated in the literature and interested readers are invited to study Refs. [35-40]. Therefore, 
we will use only a finite number of $1 / k^{2}$ (for long wave-lengths where the relation is still linear) in our calculations of interface stiffness as it will be discussed in details later.

In the present simulations, we use 1251 sampling frames over the 250 ps simulation time of the two-phase HCP-liquid coexisting slabs. The total number of frames for the time-averaging is 2502 because the calculations are averaged over the two HCP-liquid interfaces at the slab. The height of the HCP-liquid interface is located by defining a continuous order parameter $(\psi)$ to identify the HCP and liquid regions of the slab. This order parameter is defined based on the disordering of the atoms compared to their configuration in a perfect HCP crystal at the simulation temperature as

$$
\psi=\frac{\sum_{i} w_{d} r_{i} \phi_{i}}{\sum_{i} w_{d} r_{i}},
$$

where $w_{d}=\left[1-\left(\frac{r_{i}}{d}\right)^{2}\right]^{2}, r_{i}=\sqrt{\left(x_{i}-x\right)^{2}+\left(z_{i}-z\right)^{2}}, d$ is the radius of the smoothing cylinder, and the summation is over all the atoms in the smoothing distance $r_{i}<d$. Also, $\phi=(1 / 12) \sum\left|\vec{r}_{i}-\vec{r}_{H C P}\right|^{2}$, where the summation is over the twelve first nearest-neighbors, and $\vec{r}_{H C P}$ denotes the neighbor atoms position at the perfect HCPcrystal. Consequently, $\psi$ must be small in the solid state $(\sim 0.15$ here $)$ and biggerin the liquid state $(\sim 0.42$ here $)$. The interface location is determined to be the location at which $\psi$ is in between of its liquid and solid values ( $\sim 0.29$ here). For instance, Fig. 4 shows the atoms near the HCP-liquid interface for one of the sampling frames, where the atoms are colored based on the value of $\psi$ at their location, and the interface location is shown by a red curve. Once the location of the interface is determined, its time-averaged Fourier amplitudes are calculated and substituted into Eq. (5) to determine the HCP-liquid interface stiffness.

The calculated stiffness of interfaces for all the twelve slabs, along with the size and number of atoms at each slab, are listed in Table 4.As it was mentioned earlier, $b$ must be as small as possible because the general assumption in CFM is that the solid-liquid interface location is constant along the thickness direction. However, $b$ must be chosen sufficiently large to make the construction of the two-phase coexisting HCP-liquid slab possible. Also, $W$ must be chosen at least fifteen times larger than $b$ to provide sufficient data points for the calculations of 
the Fourier amplitudes. Nevertheless, we performed a convergence study on the size of the simulation slab to make sure that our interface stiffness calculations are size independent. We included the Fourier amplitudes for the first thirty modes $(k=1,2, \cdots, 30)$ in the interface stiffness calculations for all the orientations. The calculated $k_{B} T_{M} / b W\left\langle|A(k)|^{2}\right\rangle$ versus $k^{2}$ data for all the twelve orientations are depictedin Fig. 5. The stiffness of interfaces for all the orientations are obtained as the mean value of $k_{B} T_{M} / b W\left\langle|A(k)|^{2}\right\rangle$ divided by $k^{2}$, for $k=1,2, \cdots, 30$ as listed in Table 4 along with their standard statistical uncertainties. The calculated average interface stiffness for all the orientations (reported in in Table 5)is used to generate the solid lines in Fig. 5 representing the mean values of $k_{B} T_{M} / b W\left\langle|A(k)|^{2}\right\rangle$ versus $k^{2}$ data.

Table 4. The calculated interface stiffness $\left(\gamma+\gamma^{\prime \prime}\right)$ with their standard statistical uncertainties.

\begin{tabular}{lccc}
\hline \hline Interface plane & Slab size $(\mathrm{nm})$ & Number of Atoms & $\gamma+\gamma^{\prime \prime}\left(\mathrm{mJ} / \mathrm{m}^{2}\right)$ \\
\hline Basal & $1.32 \times 28.56 \times 96.20$ & 144000 & $133.4 \pm 2.2$ \\
\hline Prismatic I-a & $26.38 \times 1.60 \times 91.38$ & 153600 & $137.2 \pm 2.0$ \\
Prismatic I-b & $1.32 \times 26.72 \times 91.38$ & 128000 & $116.2 \pm 1.6$ \\
Prismatic II-a & $1.71 \times 32.07 \times 72.54$ & 158400 & $109.6 \pm 1.8$ \\
Prismatic II-b & $34.27 \times 1.60 \times 72.54$ & 158400 & $120.0 \pm 2.0$ \\
\hdashline Pyramidal I-a & $39.68 \times 1.21 \times 90.65$ & 129600 & $129.4 \pm 3.0$ \\
Pyramidal I-b & $1.32 \times 31.51 \times 90.65$ & 149760 & $117.4 \pm 2.0$ \\
Pyramidal II-a & $31.41 \times 1.26 \times 105.77$ & 165880 & $117.6 \pm 1.6$ \\
Pyramidal II-b & $1.71 \times 29.51 \times 97.63$ & 196272 & $114.6 \pm 2.8$ \\
Twinning I & $1.32 \times 31.29 \times 105.31$ & 172800 & $117.6 \pm 3.2$ \\
Twinning II & $1.14 \times 33.56 \times 108.69$ & 165600 & $117.8 \pm 2.8$ \\
Twinning III & $1.32 \times 30.29 \times 113.29$ & 180000 & $121.0 \pm 3.2$ \\
\hline
\end{tabular}

From Fig. 5, it is clear that most of the presented data are fairly close to their related mean lines, which demonstrates the accuracy of the calculations. The standard statistical uncertainties for interface stiffness calculations are $\sim 2$ to $3 \mathrm{~mJ} / \mathrm{m}^{2}$. Another significant highlight of the current calculations is the large number of Fourier modes (30 modes) used in the calculations which is much more than the number of Fourier modes in the previous works [6]. This is mainly owed to the new order parameter $\psi$ which damps the fluctuations of the previously used order parameter $(\phi)$. However, using of order parameter $\psi$ in CFM requires determinationof the smoothing distance, $d$, which implies a further computational work. We 
determined $d$ for each orientation such that: a) the calculated interface stiffness is converged, and $b$ ) the resulted standard statistical uncertainty is minimum.

\subsection{Interface free energy and surface anisotropy}

Equatingthe relations for the interface stiffness determined by HCP symmetry-adapted spherical harmonics (the fourth column of Table 3) with the corresponding calculations of the interface stiffness by MD simulations (the last column of Table 4) results in twelve equations to solve for $\gamma_{0}, \varepsilon_{20}, \varepsilon_{40}, \varepsilon_{60}$, and $\varepsilon_{66}$. From the twelve resultant equations, the equations related to Prismatic I-a, I-b, II-a, and II-b need an especial attention. By adding/subtracting the interface stiffness equation related to Prismatic I-a and I-bto equations related to Prismatic II-a and II-b, respectively, these equations can be written as

$$
\begin{aligned}
& \gamma_{0}\left[1-\frac{1}{4} \sqrt{\frac{5}{\pi}} \bar{\varepsilon}_{20}+\frac{9}{16} \sqrt{\frac{1}{\pi}} \bar{\varepsilon}_{40}-\frac{5}{32} \sqrt{\frac{13}{\pi}} \bar{\varepsilon}_{60}\right]=123.4 \\
& -\gamma_{0} \frac{35}{64} \sqrt{\frac{6006}{\pi}} \bar{\varepsilon}_{66}=6.9 \\
& \gamma_{0}\left[1+\frac{5}{4} \sqrt{\frac{5}{\pi}} \bar{\varepsilon}_{20}-\frac{171}{16} \sqrt{\frac{1}{\pi}} \bar{\varepsilon}_{40}+\frac{205}{32} \sqrt{\frac{13}{\pi}} \bar{\varepsilon}_{60}\right]=118.1 \\
& -\gamma_{0} \frac{5}{64} \sqrt{\frac{6006}{\pi}} \bar{\varepsilon}_{66}=0.95
\end{aligned}
$$

It is obvious that only three of these four equations are independent. Therefore, including the equations related for at least one other orientation is necessary (other than the equations related to Basal, Prismatic I-a, I-b, II-a, and II-b) to determine $\gamma_{0}, \varepsilon_{20}, \varepsilon_{40}, \varepsilon_{60}$, and $\varepsilon_{66}$ .ConsideringEqs. (7b) and (7d) simultaneously makes it clear that the anisotropy parameter $\varepsilon_{66}$ is governed by the difference between the stiffness of interfaces for Prismatic I-a and II-a, or I-b and II-b. Furthermore, $\gamma_{0} \bar{\varepsilon}_{66}(5 / 64) \sqrt{6006 / \pi}$ is equal to0.986 or0.95 as a result of Eq. (7b) or (7d), respectively; the resultant error in this calculation is considered a reasonable error in the concept of CFM and MD simulation.

To calculate $\gamma_{0}, \varepsilon_{20}, \varepsilon_{40}, \varepsilon_{60}$, and $\varepsilon_{66}$, we formed fourteen system of equations, each consisting of five equations. In each system of equations, we included the Basal equation, Eqs. (7a) and (7c), Eq. (7b) or (7d), and one of the remaining seven equations related to the other 
orientations. The calculated mean value of $\gamma_{0}, \varepsilon_{20}, \varepsilon_{40}, \varepsilon_{60}$, and $\varepsilon_{66}$ along with their standard statistical uncertainties are reported in Table 5.

Table 5. The average HCP-liquid interface free energy $\gamma_{0}$ and anisotropy parameters $\varepsilon_{20}, \varepsilon_{40}$, $\varepsilon_{60}$, and $\varepsilon_{66}$ for $\mathrm{Mg}$.

\begin{tabular}{lccccc}
\hline \hline Potential & $\gamma_{0}\left(m J / m^{2}\right)$ & $\varepsilon_{20}(\%)$ & $\varepsilon_{40}(\%)$ & $\varepsilon_{60}(\%)$ & $\varepsilon_{66}(\%)$ \\
\hline MEAM; present & $122.2 \pm 0.1$ & $-3.45 \pm 0.34$ & $-0.47 \pm 0.04$ & $-0.06 \pm 0.03$ & $-0.46 \pm 0.01$ \\
EAM; [6] & $89.9 \pm 1.5$ & $-2.6 \pm 1.5$ & --- & --- & $0.3 \pm 0.1$ \\
\hline
\end{tabular}

Also, the HCP-liquid interface stiffness and energy for different orientations are calculated by substituting these calculated parameters into the relations in the third and fourth columns of Table 3, respectively. The calculated values are listed in Table 6 and labeled as "Fitted." All the fitted calculations of interface stiffness for different casesare in agreement with the MD calculations, with minimal differences which are in the range of the standard statistical uncertainties of the MD calculated data. Moreover, the HCP-liquid interface free energy for the closed packed plane (Basal orientation) is minimum similar to the previous findings for $\mathrm{Mg}$ [6] and for FCC metals, where $\gamma_{111}$ found to be minimum [19]. It is worthy to note that after the Basal orientation, the next minimum interface free energybelongs to the Twinning III orientation, and not to the planes with higher symmetries, such asPrismatic planes.

To the knowledge of the authors, there are no experimental measurements of $\gamma_{0}$ and anisotropy parameters for Mg. However, $\gamma_{0}$ has been experimentally measured for other HCP elements like $\mathrm{Zn}[41,42]$ and $\mathrm{Cd}[43,44]$. Considering the previously proven concept that $\gamma_{0}$ for a material is directly related to its melting point and latent heat [19], a qualitative comparison of the calculated $\gamma_{0}$ for $\mathrm{Mg}$ with the $\gamma_{0}$ for other $\mathrm{HCP}$ elements is possible. Between $\mathrm{Zn}$ and $\mathrm{Cd}$, the melting point and latent heat of $\mathrm{Zn}$ is closer to $\mathrm{Mg}$. The experimental measurements of $\gamma_{0}$ for $\mathrm{Zn}$ are 77 and $87 \pm 15\left(\mathrm{~mJ} / \mathrm{m}^{2}\right)$ depending on the experimental method. Since the melting point and latent heat of $\mathrm{Zn}$ is, respectively, 1.33 and 1.16 timeslowerthan those of $\mathrm{Mg}$, it is expected that $\gamma_{0}$ for $\mathrm{Mg}$ be at least higher than $\gamma_{0}$ of $\mathrm{Zn}$, which is in agreement with our calculations. Sun et al. [6] calculated $\gamma_{0}, \varepsilon_{20}, \varepsilon_{40}, \varepsilon_{60}$, and $\varepsilon_{66}$ for Mg using CFM and EAM potential. Those 
results are also listed in the third row of Table 5 for comparison. They only considered the first five orientations (Basal, Prismatic I-a, I-b, II-a, and II-b) in their interface stiffness calculations which will produce four independent equations, as it was mentioned earlier. Therefore, they determined $\gamma_{0}, \varepsilon_{20}$, and $\varepsilon_{66}$ assuming that $\varepsilon_{40}$ and $\varepsilon_{60}$ are zero. However, they argued that including $\varepsilon_{40}$ is necessary to get the experimentally observed grain growth direction in $\mathrm{Mg}$ alloys, and $\varepsilon_{66}$ may be positive or negative. The grain growth direction predictions will be discussed in details in the next section. Nevertheless, we determine the anisotropy parameters $\varepsilon_{20}$ and $\varepsilon_{66}$ to be in the order of Sun and coworkers calculations; i.e., our calculations are, respectively, $33 \%$ and $53 \%$ higher but with much less uncertainties.

Table 6. The HCP-liquid interface stiffness $\left(\gamma+\gamma^{\prime \prime}\right)$ and free energy $(\gamma)$ as calculated by fitting to the HCP symmetry-adaptedspherical harmonics compared to the MD calculated interface stiffness.

\begin{tabular}{cccc}
\hline \hline \multirow{2}{*}{ Interface plane } & \multicolumn{2}{c}{$\gamma+\gamma^{\prime \prime}\left(\mathrm{mJ} / \mathrm{m}^{2}\right)$} & $\gamma\left(\mathrm{mJ} / \mathrm{m}^{2}\right)$ \\
\cline { 2 - 4 } & MD & Fitted & Fitted \\
\hline Basal & $133.4 \pm 2.2$ & 133.4 & 119.0 \\
\hdashline Prismatic I-a & $137.2 \pm 2.0$ & 137.0 & 123.0 \\
Prismatic I-b & $116.2 \pm 1.6$ & 116.2 & 123.8 \\
Prismatic II-a & $109.6 \pm 1.8$ & 109.9 & 123.8 \\
Prismatic II-b & $120.0 \pm 2.0$ & 120.0 & 123.0 \\
\hdashline Pyramidal I-a & $129.4 \pm 3.0$ & 129.2 & 122.6 \\
Pyramidal I-b & $117.4 \pm 2.0$ & 116.8 & 122.6 \\
Pyramidal II-a & $117.6 \pm 1.6$ & 117.5 & 122.8 \\
Pyramidal II-b & $114.6 \pm 2.8$ & 116.3 & 122.7 \\
\hline Twinning I & $117.6 \pm 3.2$ & 118.6 & 121.7 \\
Twinning II & $117.8 \pm 2.8$ & 118.6 & 122.9 \\
Twinning III & $121.0 \pm 3.2$ & 120.0 & 120.7 \\
\hline
\end{tabular}

\subsection{Dendrite growth direction and rate}


The anisotropy of the solid-liquid interface free energy can be used to predict the preferred dendrite growth directions and rates by considering Gibbs-Thompson relation for the local solid-liquid interface equilibrium temperature $(T)$ as[45]

$$
T=T_{M}+m C_{L}-\frac{T_{M}}{L} \sum_{i=1}^{2}\left(\gamma(\hat{\mathrm{n}})+\frac{\partial^{2} \gamma(\hat{\mathrm{n}})}{\partial \theta_{i}^{2}}\right) K_{i}
$$

where $T_{M}$ is the melting temperature, $m$ is the liquidus slope, $C_{L}$ is the solute composition at the liquid interface, $L$ is the latent heat, $K_{i}$ are the mean curvatures of the interface, $\theta_{i}$ are the local angles between the normal direction and local principal directions, and $\gamma(\hat{\mathrm{n}})+\frac{\partial^{2} \gamma(\hat{\mathrm{n}})}{\partial \theta_{i}^{2}}$ is the interface stiffness. Eq. (8) implies that the preferred dendrite direction is the direction at which the interface stiffness is minimum, and the dendrite growth rate is related to the inverse of the interface stiffness. Although the interface stiffness in Eq. (8) is a tensor in three dimensions, the trace of the interface stiffness tensor $(\operatorname{Tr} S)$ can approximately predict the dendrite growth direction and rate [6], as

$$
\operatorname{Tr} S=2 \gamma+\frac{\partial^{2} \gamma}{\partial \theta^{2}}+\frac{1}{\sin ^{2} \theta} \frac{\partial^{2} \gamma}{\partial \phi^{2}}+\frac{\cos \theta}{\sin \theta} \frac{\partial \gamma}{\partial \theta}
$$

where therelation for $\gamma$ in terms of HCPsymmetry-adaptedspherical harmonics is given in Eq. (3). The plot of $1 / \operatorname{Tr} S$ in spherical coordinates is shown in Fig. 6, where the anisotropy parameters in $\gamma$ aresubstituted from the present MD data (Table 5). The preferred dendrite growth directions are the directions at which $1 / \operatorname{Tr} S$ are locally maximum. Our calculations show that the absolute maximum of $1 / \operatorname{Tr} S$ occurs at the basal plane and $[11 \overline{2} 0]$ direction, and this is the primary preferred growth direction (primary dendrite arms direction). Also, the absolute minimum and the least preferred growth direction is [0001]. These findings are in agreement with the EAM-MD calculations of Sun at al. [6] who calculated the anisotropy parameters as given in the third row of Table 5.In addition, we calculated a local maximum approximately in [33 $\overline{6} 2]$ direction and a local minimum in[ $\overline{1} 100]$ direction, where the absolute value of $1 / \operatorname{Tr} S$ in [33 $\overline{6} 2]$ direction is bigger than its value in [ $\overline{1} 100]$ direction. Therefore, [33 $\overline{6} 2]$ is the second preferred dendrite growth direction for $\mathrm{Mg}$. There is no published experimental work on determining the dendrite growth directions for elemental $\mathrm{Mg}$. The only related work is the work 
of Pettersen et al. [46] who used Bridgman equipment to conduct a directional solidification slab experiment for low gradient to velocity ratiosolidification for AZ91 alloy. They observed [1120] as the primary dendrite growth direction similar to our prediction for elemental $\mathrm{Mg}$. They also observed four secondary dendrite arms at[224̄5] crystallographic direction for AZ91, while we predicted four secondary dendrite arms all approximately in [33 $\overline{6} 2$ ] direction for elemental $\mathrm{Mg}$. More computational works using the present MEAM-MD and CFM method are required to study the variation of the dendrite growth directions of $\mathrm{Mg}$ by adding $9 \%$ weight of $\mathrm{Al}$ and $1 \%$ weight of $\mathrm{Zn}$ to form AZ91 alloy.

\section{Conclusions}

A comprehensive study was performed to determine HCP-liquid interface free energy and anisotropy for the case study of Mg. In the concept of CFM, MEAM interatomic potentials were used in the MD simulations, and the pyramidal and twinning (as well as basal and prismatic) orientations were considered for the first time to determine the HCP-liquid interface

properties. The average interface free energy was calculated to be $\gamma_{0}=122.2 \pm 0.1 \mathrm{~mJ} / \mathrm{m}^{2}$, and the HCP-liquid interface anisotropic parameters were calculated to be $\varepsilon_{20}=-3.45 \pm 0.34$, $\varepsilon_{40}=-0.47 \pm 0.04, \varepsilon_{66}=-0.46 \pm 0.01$, and a negligible $\varepsilon_{60}$. The uncertainty involved with these calculations was less than $2 \%$ which was achieved because ofemploying a continuous order parameter to accurately locate the HCP-liquid interface. Furthermore, the calculations of the HCP-liquid interface free energy and anisotropy predicts the primary dendrite growth direction of $[11 \overline{2} 0]$ and a secondary dendrite growth direction of $[33 \overline{6} 2]$.

\section{Acknowledgments}

The authors are grateful for computer time allocation provided by the Extreme Science and Engineering Discovery Environment (XSEDE). M. Asle Zaeem would like to acknowledge the funding support from the National Science Foundation under Grant No. NSF-CMMI 1537170.

References 
[1] W. Curtin. Density-functional theory of the solid-liquid interface, Physical review letters 59 (1987) 1228.

[2] J. Hoyt, M. Asta, A. Karma. Method for computing the anisotropy of the solid-liquid interfacial free energy, Physical review letters 86 (2001) 5530.

[3] J.Q. Broughton, G.H. Gilmer. Molecular dynamics investigation of the crystal-fluid interface. VI. Excess surface free energies of crystal-liquid systems, The Journal of chemical physics 84 (1986) 57595768.

[4] R.L. Davidchack, B.B. Laird. Direct calculation of the hard-sphere crystal/melt interfacial free energy, Physical review letters 85 (2000) 4751.

[5] R.L. Davidchack, B.B. Laird. Direct calculation of the crystal-melt interfacial free energies for continuous potentials: Application to the Lennard-Jones system, The Journal of chemical physics 118 (2003) 7651-7657.

[6] D. Sun, M. Mendelev, C. Becker, K. Kudin, T. Haxhimali, M. Asta, J. Hoyt, A. Karma, D. Srolovitz. Crystal-melt interfacial free energies in hcp metals: A molecular dynamics study of Mg, Physical Review B 73 (2006) 024116.

[7] J. Hoyt, M. Asta. Atomistic computation of liquid diffusivity, solid-liquid interfacial free energy, and kinetic coefficient in Au and Ag, Physical Review B 65 (2002) 214106.

[8] J. Hoyt, M. Asta, A. Karma. Atomistic and continuum modeling of dendritic solidification, Materials Science and Engineering: R: Reports 41 (2003) 121-163.

[9] D. Sun, M. Asta, J. Hoyt. Crystal-melt interfacial free energies and mobilities in fcc and bcc Fe, Physical Review B 69 (2004) 174103.

[10] M.S. Daw, M.I. Baskes. Embedded-atom method: Derivation and application to impurities, surfaces, and other defects in metals, Physical Review B 29 (1984) 6443.

[11] P. Bavli, J. Adler. Parallel codes for simulating elastic constants and melting in Ar and Mg, Physics Procedia 4 (2010) 3-7.

[12] P. Bavli, E. Polturak, J. Adler. Molecular dynamics study of melting of the hcp metal Mg, Physical Review B 84 (2011) 235442.

[13] P. Bavli, M. Sc. Melting in HCP Lattices. Technion-Israel Institute of Technology, Faculty of Physics, 2009.

[14] Y. Bai. MOLECULAR DYNAMICS SIMULATION STUDY OF SOLID-LIQUID INTERFACE PROPERTIES OF HCP MAGNESIUM, (2012).

[15] Y. Gao, Y. Yang, D. Sun, M. Asta, J. Hoyt. Molecular dynamics simulations of the crystal-melt interface mobility in HCP Mg and BCC Fe, Journal of Crystal Growth 312 (2010) 3238-3242.

[16] Z. Xia, D. Sun, M. Asta, J. Hoyt. Molecular dynamics calculations of the crystal-melt interfacial mobility for hexagonal close-packed Mg, Physical Review B 75 (2007) 012103.

[17] M. Baskes. Modified embedded-atom potentials for cubic materials and impurities, Physical Review B 46 (1992) 2727.

[18] B. Jelinek, S. Groh, M. Horstemeyer, J. Houze, S. Kim, G. Wagner, A. Moitra, M. Baskes. Modified embedded atom method potential for Al, Si, Mg, Cu, and Fe alloys, Physical Review B 85 (2012) 245102.

[19] E. Asadi, M.A. Zaeem, S. Nouranian, M.I. Baskes. Two-phase solid-liquid coexistence of Ni, Cu, and $\mathrm{Al}$ by molecular dynamics simulations using the modified embedded-atom method, Acta Materialia 86 (2015) 169-181.

[20] E. Asadi, M.A. Zaeem, S. Nouranian, M.I. Baskes. Quantitative modeling of the equilibration of two-phase solid-liquid Fe by atomistic simulations on diffusive time scales, Physical Review B 91 (2015) 024105.

[21] Y.-M. Kim, N.J. Kim, B.-J. Lee. Atomistic modeling of pure $\mathrm{Mg}$ and $\mathrm{Mg}-\mathrm{Al}$ systems, Calphad 33 (2009) 650-657. 
[22] B.-J. Lee, M. Baskes. Second nearest-neighbor modified embedded-atom-method potential, Physical Review B 62 (2000) 8564.

[23] B.-J. Lee, M. Baskes, H. Kim, Y.K. Cho. Second nearest-neighbor modified embedded atom method potentials for bcc transition metals, Physical Review B 64 (2001) 184102.

[24] S. Nouranian, M.A. Tschopp, S.R. Gwaltney, M.I. Baskes, M.F. Horstemeyer. An Interatomic Potential for Saturated Hydrocarbons Based on the Modified Embedded-Atom Method, Physical Chemistry Chemical Physics 16 (2014) 6233-6249.

[25] S. Plimpton. Fast parallel algorithms for short-range molecular dynamics, Journal of Computational Physics 117 (1995) 1-19.

[26] A. Luque, M. Ghazisaeidi, W. Curtin. Deformation modes in magnesium $\left(\begin{array}{llll}0 & 0 & 0 & 1\end{array}\right)$ and $\$\left(\begin{array}{ll}0 & 1 \backslash \backslash\end{array}\right.$ bar $\{1\} \backslash 1)$ \$ single crystals: simulations versus experiments, Modelling and Simulation in Materials Science and Engineering 21 (2013) 045010.

[27] E. Asadi, M.A. Zaeem, M.I. Baskes. Phase-Field Crystal Model for Fe Connected to MEAM Molecular Dynamics Simulations, JOM 66 (2014) 429-436.

[28] S. Nosé. A unified formulation of the constant temperature molecular dynamics methods, The Journal of Chemical Physics 81 (1984) 511.

[29] W.G. Hoover. Canonical dynamics: Equilibrium phase-space distributions, Physical Review A 31 (1985) 1695.

[30] R. Rozas, J. Horbach. Capillary wave analysis of rough solid-liquid interfaces in nickel, EPL (Europhysics Letters) 93 (2011) 26006.

[31] D.A. Varshalovich, A. Moskalev, V. Khersonskii. Quantum theory of angular momentum, World Scientific, 1988.

[32] M. Kara, K. Kurki-Suonio. Symmetrized multipole analysis of orientational distributions, Acta Crystallographica Section A: Crystal Physics, Diffraction, Theoretical and General Crystallography 37 (1981) 201-210.

[33] M.P. Fisher, D.S. Fisher, J.D. Weeks. Agreement of capillary-wave theory with exact results for the interface profile of the two-dimensional Ising model, Physical Review Letters 48 (1982) 368.

[34] D.S. Fisher, J.D. Weeks. Shape of crystals at low temperatures: Absence of quantum roughening, Physical review letters 50 (1983) 1077.

[35] F. Schmitz, P. Virnau, K. Binder. Logarithmic finite-size effects on interfacial free energies: Phenomenological theory and Monte Carlo studies, Physical Review E 90 (2014) 012128.

[36] J.J. Morris. Finite-Size scaling of the interfacial tension, Journal of statistical physics 69 (1992) 539-571.

[37] M.P. Gelfand, M.E. Fisher. Finite-size effects in fluid interfaces, Physica A: Statistical Mechanics and its Applications 166 (1990) 1-74.

[38] V. Privman. Finite-size properties of the angle-dependent surface tension of rough interfaces, Physical review letters 61 (1988) 183.

[39] D. Abraham, N. Švrakić. Exact finite-size effects in surface tension, Physical review letters 56 (1986) 1172.

[40] E. Brézin, J. Zinn-Justin. Finite size effects in phase transitions, Nuclear Physics B 257 (1985) 867-

893.

[41] D.W.G. White. Transactions of the Metallurgical Society of AIME 236 (1966) 796.

[42] A. Passerone, R. Sangiorgi, N. Eustathopoulos, P. Desre. Microstructure and interfacial tensions in Zn-In and Zn-Bi alloys, Metal Science 13 (1979) 359-365.

[43] K. Kelton. Crystal nucleation in liquids and glasses, Solid state physics 45 (1991) 75-177.

[44] D. WHITE. SURFACE TENSIONS OF IN AND CD, METALL TRANS 3 (1972).

[45] R.W. Cahn, P. Haasen. Physical metallurgy. Vol. 1, North-Holland, 1996. 
[46] K. Pettersen, O. Lohne, N. Ryum. Dendritic solidification of magnesium alloy AZ91, Metallurgical Transactions A 21 (1990) 221-230. 


\section{Figures Caption:}

Fig. 1. The schematic of the eight different HCP-liquid interface planes.

Fig. 2. The schematic of the two-phase solid-liquid coexisting slab used in CFM.

Fig. 3. Plots of HCP-symmetry adopted spherical harmonics, a) $y_{20}$, b) $y_{40}$, c) $y_{60}$, and d) $y_{66}$.

Fig. 4. A snapshot of the two-phase HCP-liquid coexisting slab of $\mathrm{Mg}$ near the interface.The upper half is solid, the lower half is liquid, and the red curve shows the location of the HCPliquid interface.

Fig. 5.The plot of $k_{B} T_{M} / b W\left\langle|A(k)|^{2}\right\rangle$ versus $k^{2}$ data where the lines are the mean values and the interface planes are: a) Basal, b) Prismatic I, c) Prismatic II, d) Pyramidal I, e) Pyramidal II, and f) Twinning I, II, and III.

Fig. 6.The plot of $1 / \operatorname{Tr} S$ in spherical coordinate system where the anisotropy parameters are substituted from the present MD data (Table 5). 

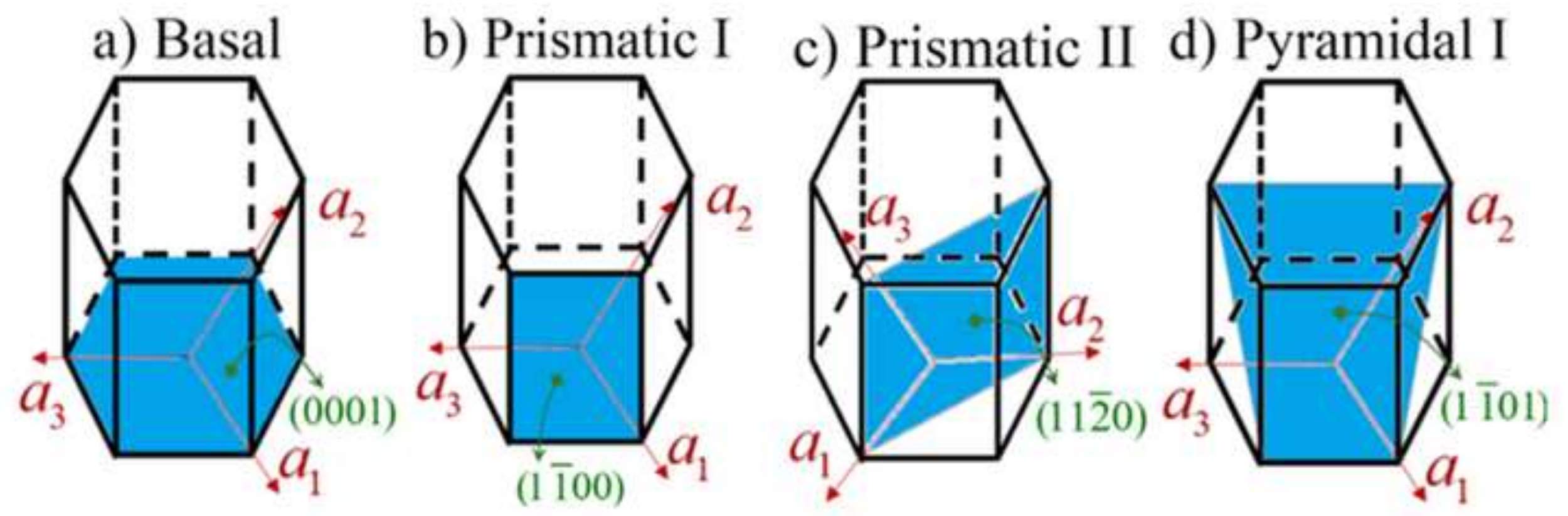

e) Pyramidal II f ) Twinning I
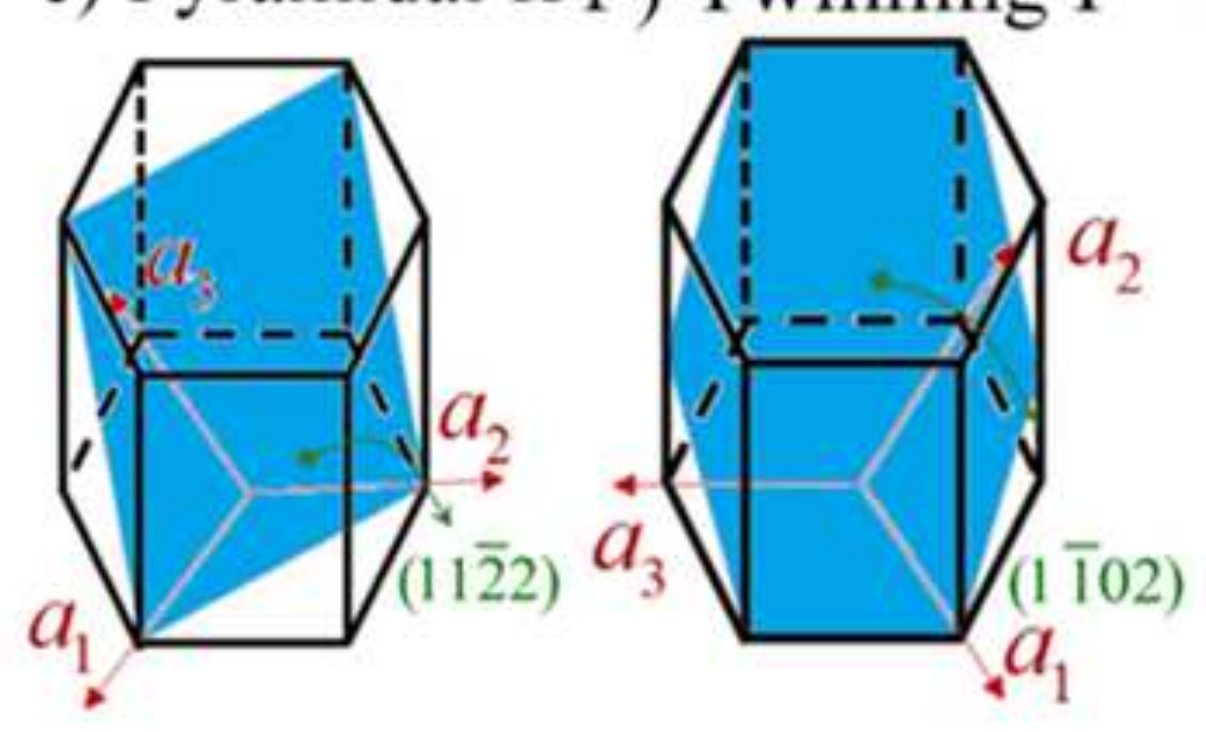

g) Twinning II

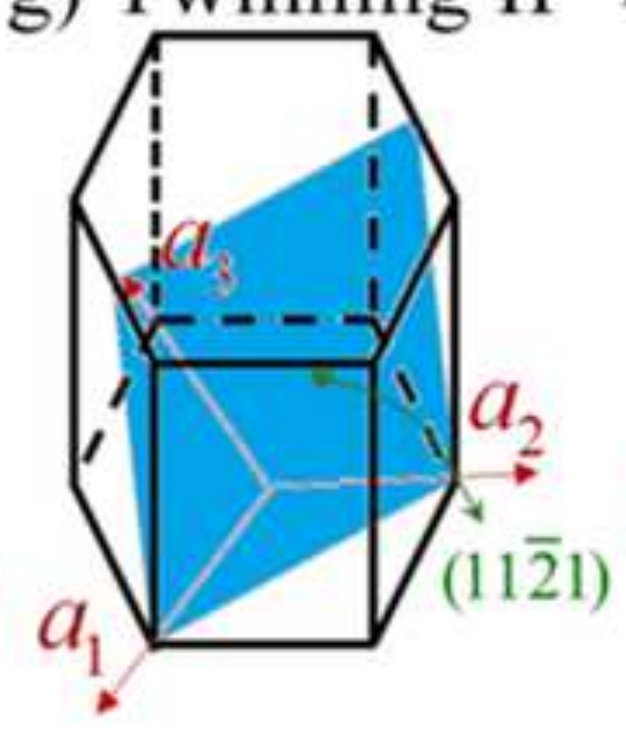

h) Twinning III

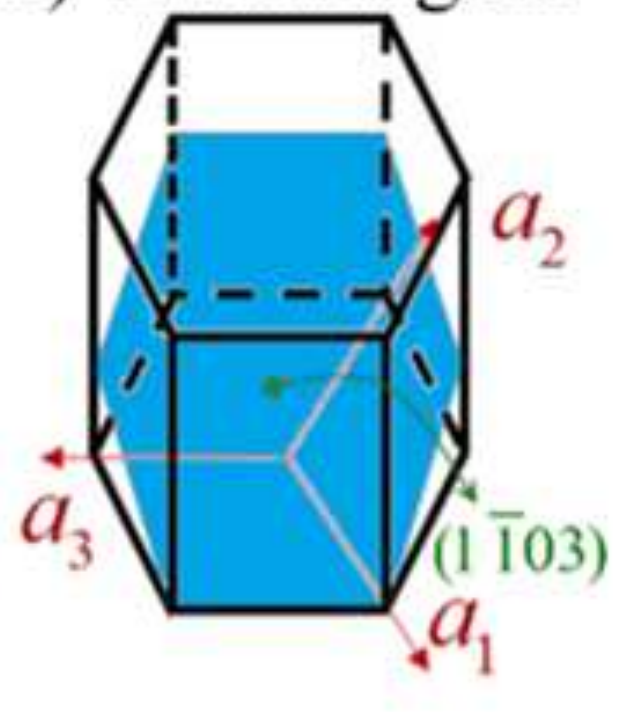




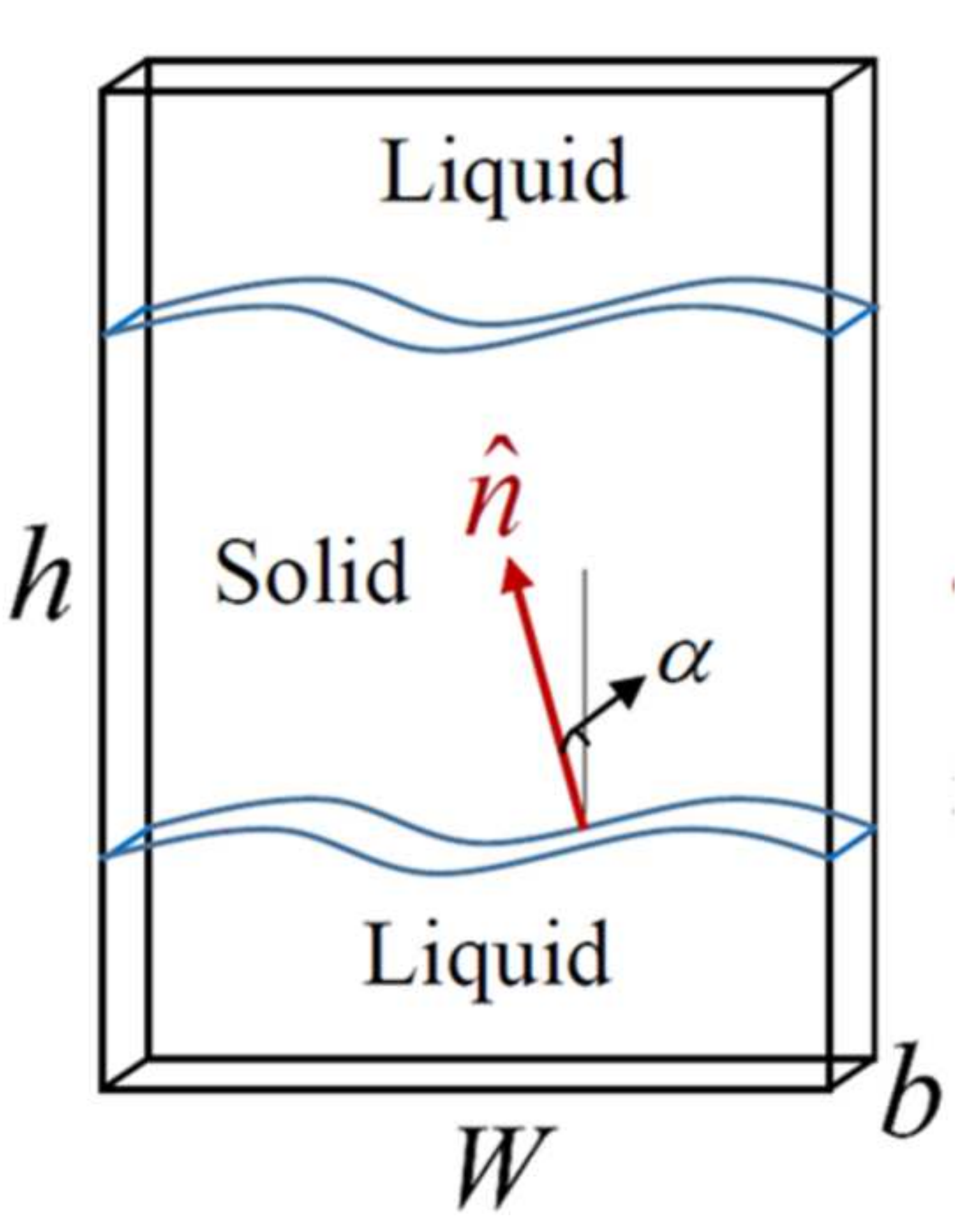

Figure 2

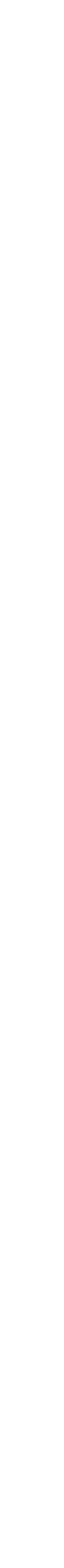

\section{Figure}
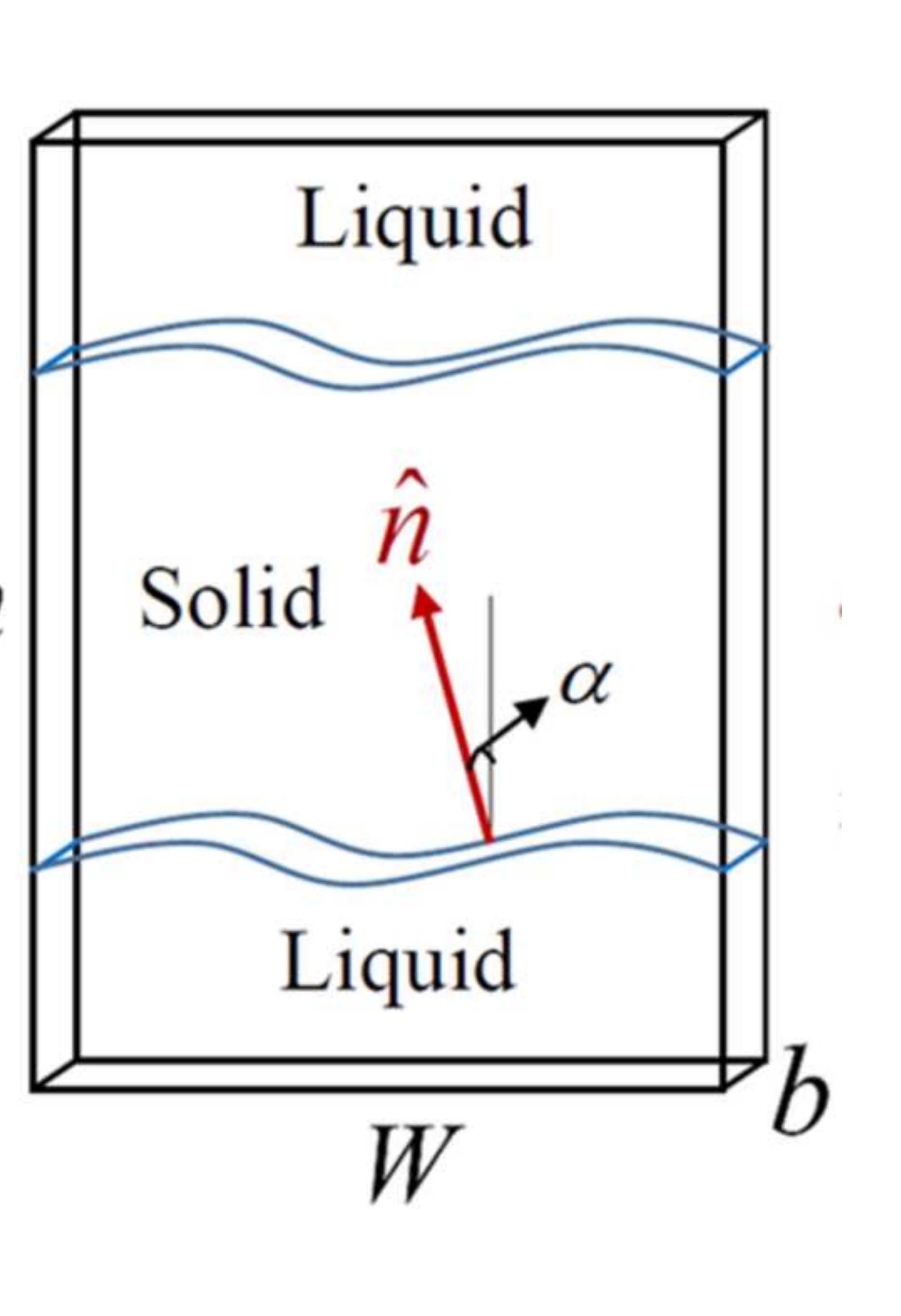
Figure 3

a) $y_{20}(\theta, \phi)$

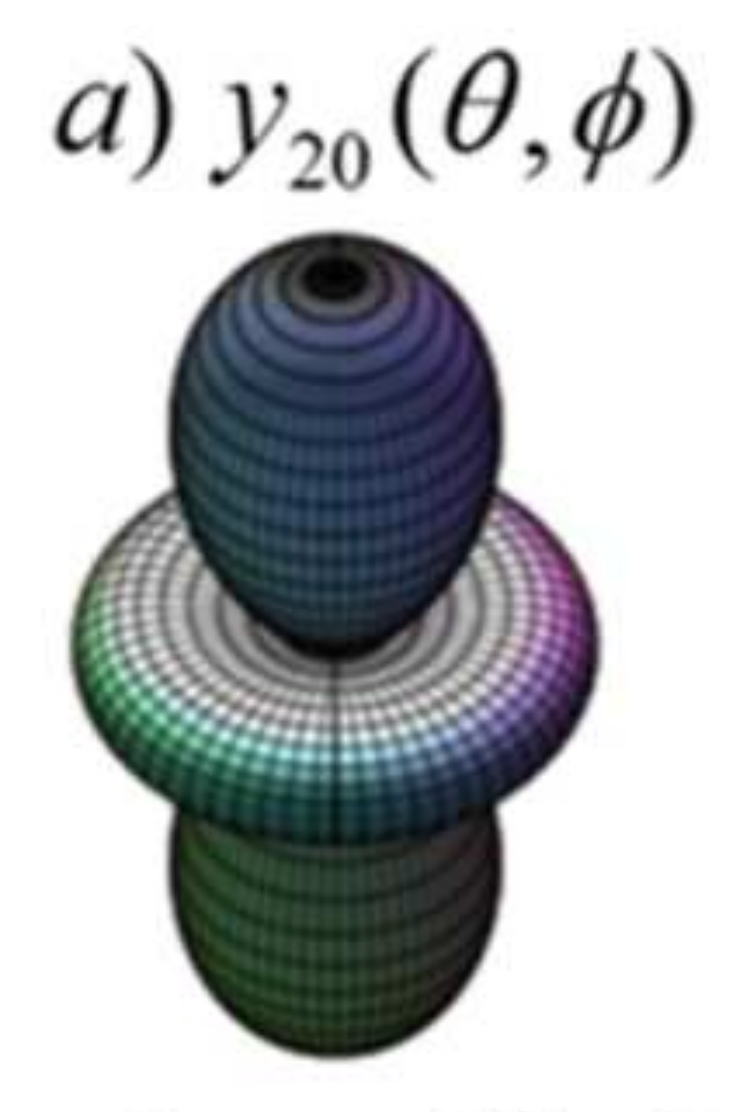

c) $y_{60}(\theta, \phi)$
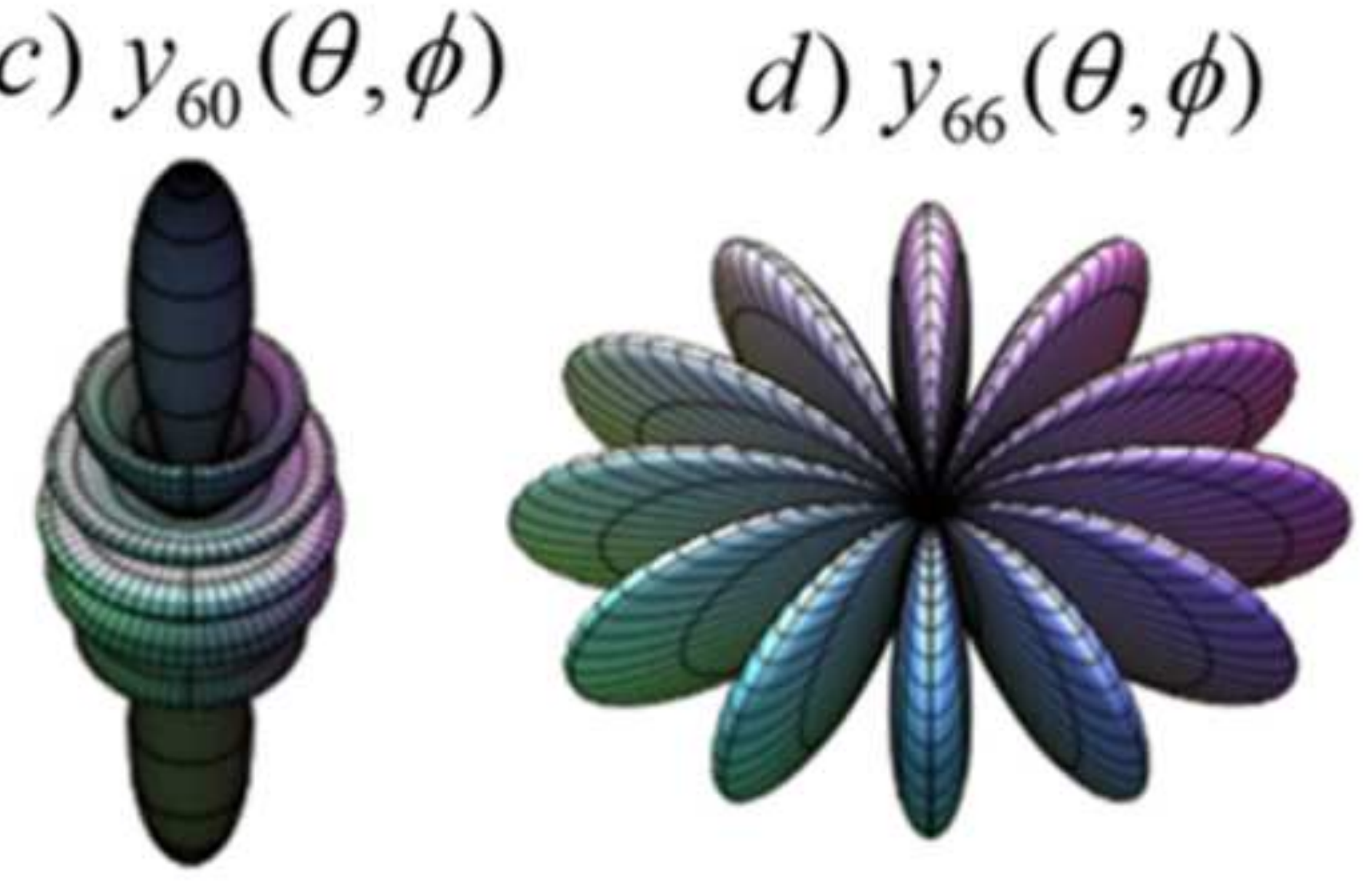

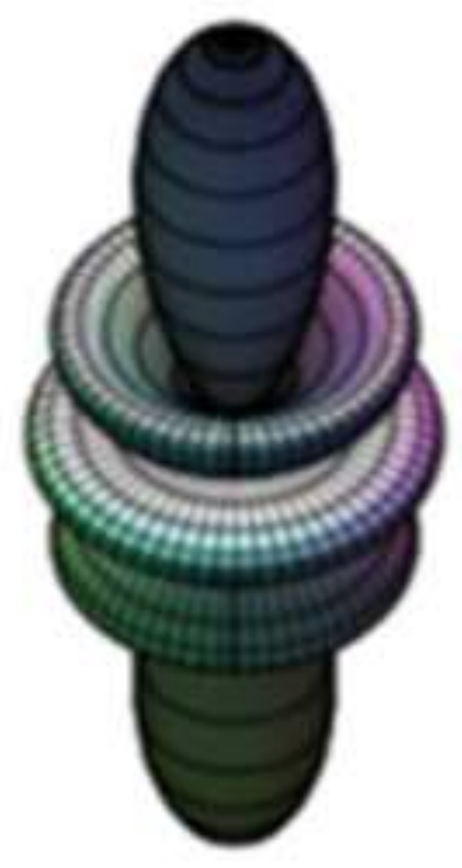

d) $y_{66}(\theta, \phi)$

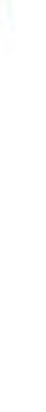

b) $y_{40}(\theta, \phi)$

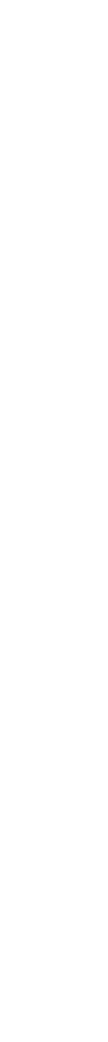

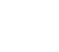
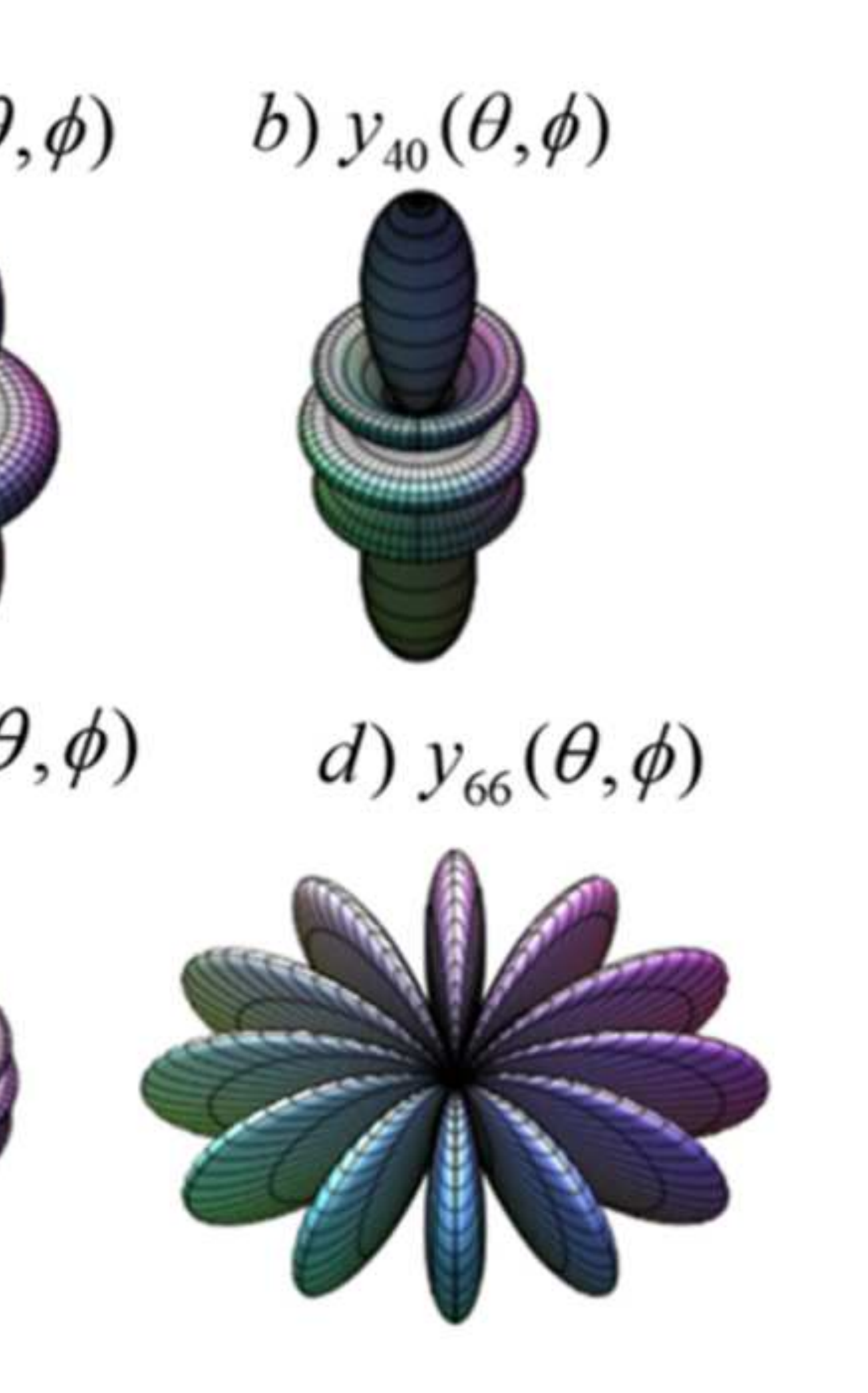

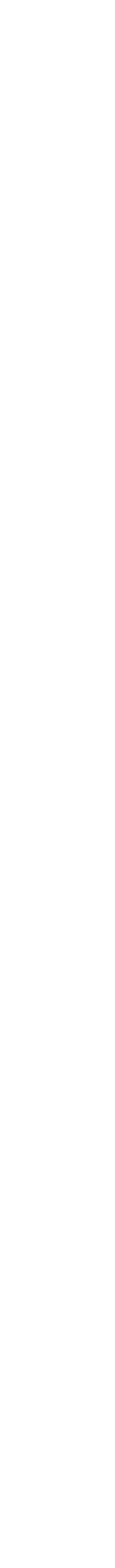

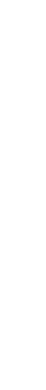




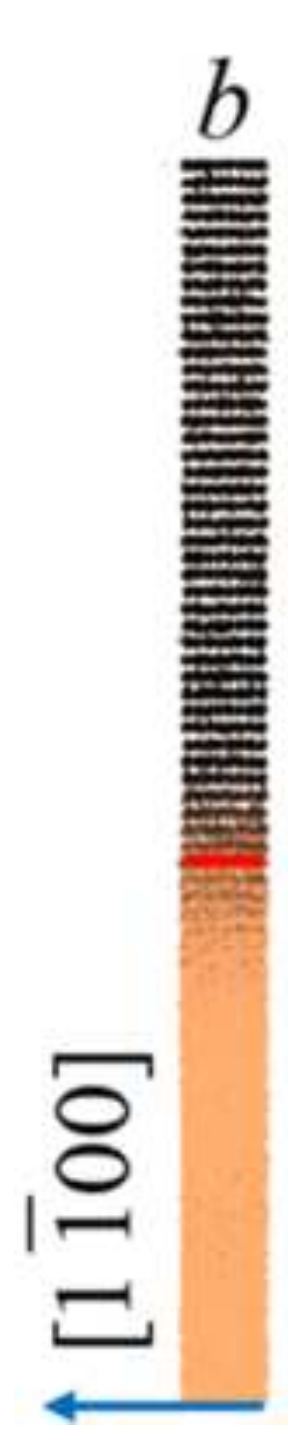

$\uparrow[0001] \quad W$

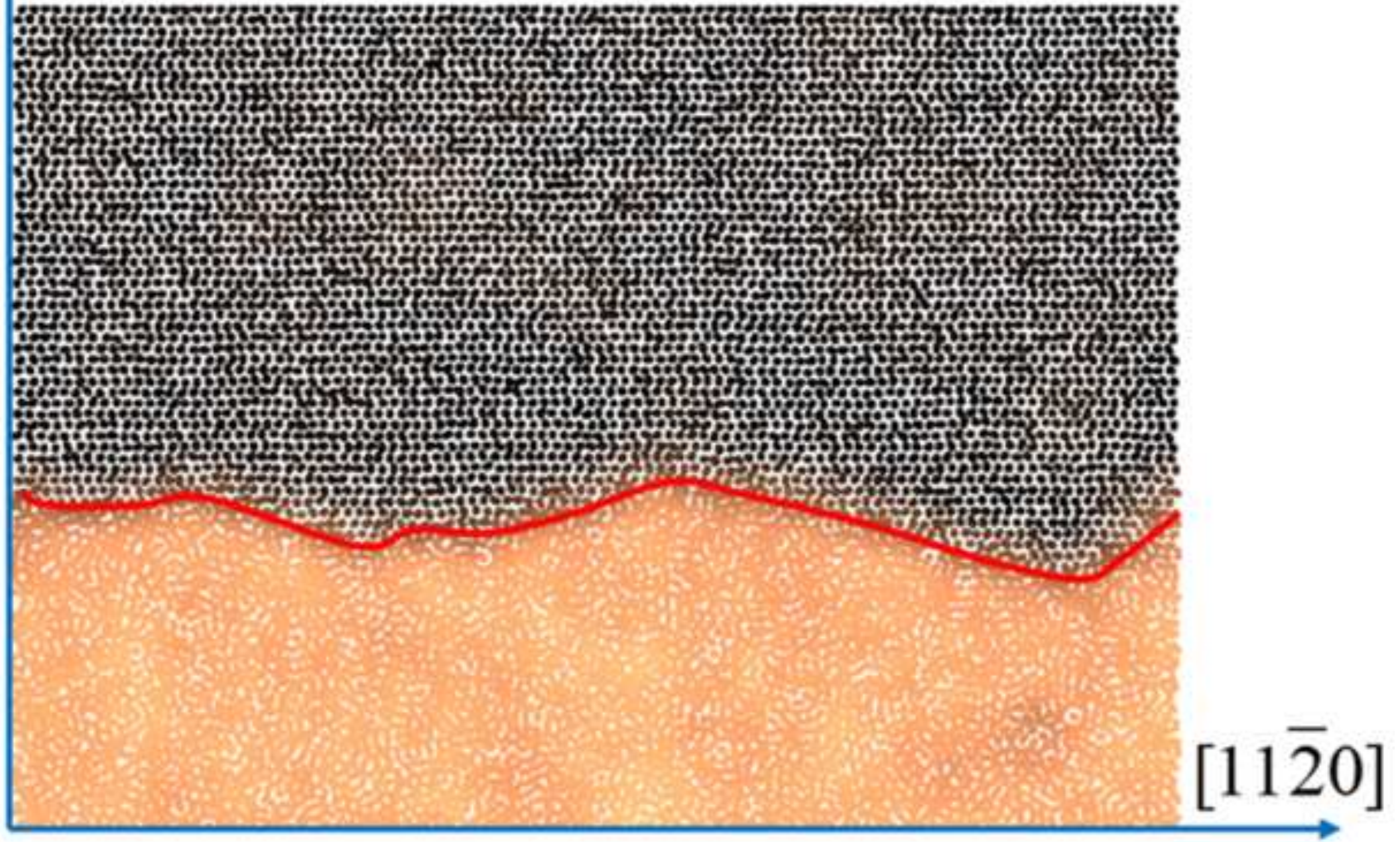



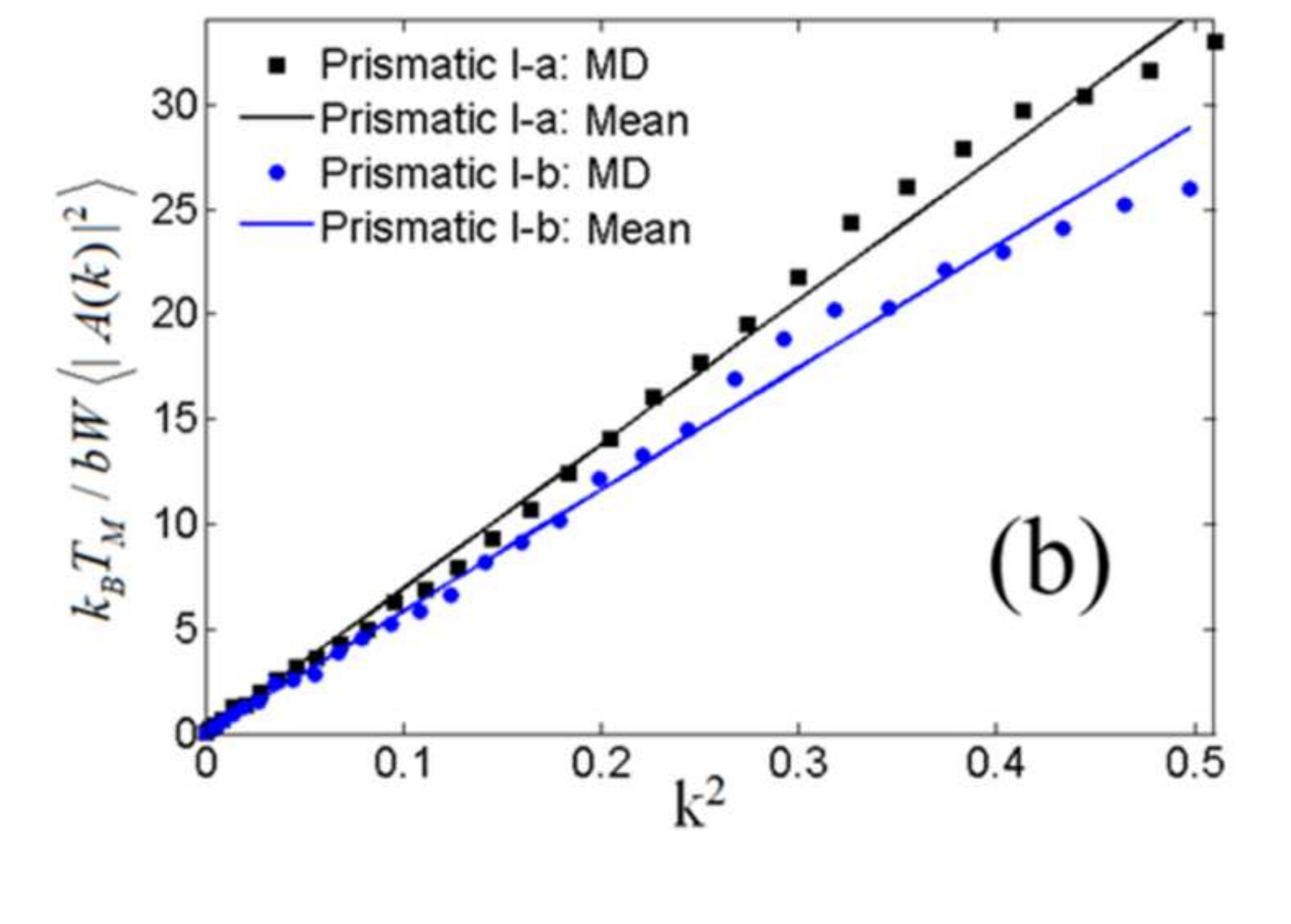

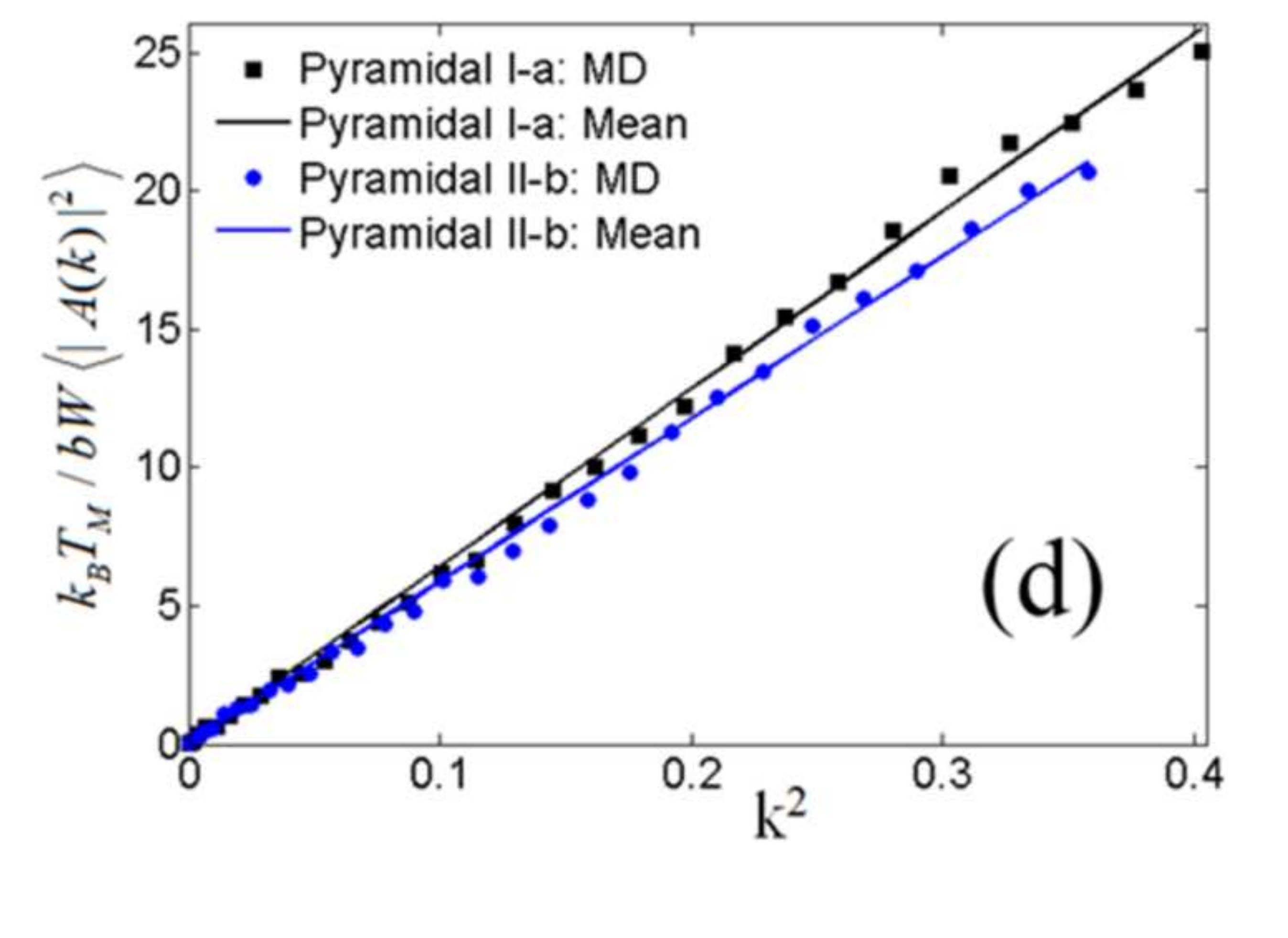

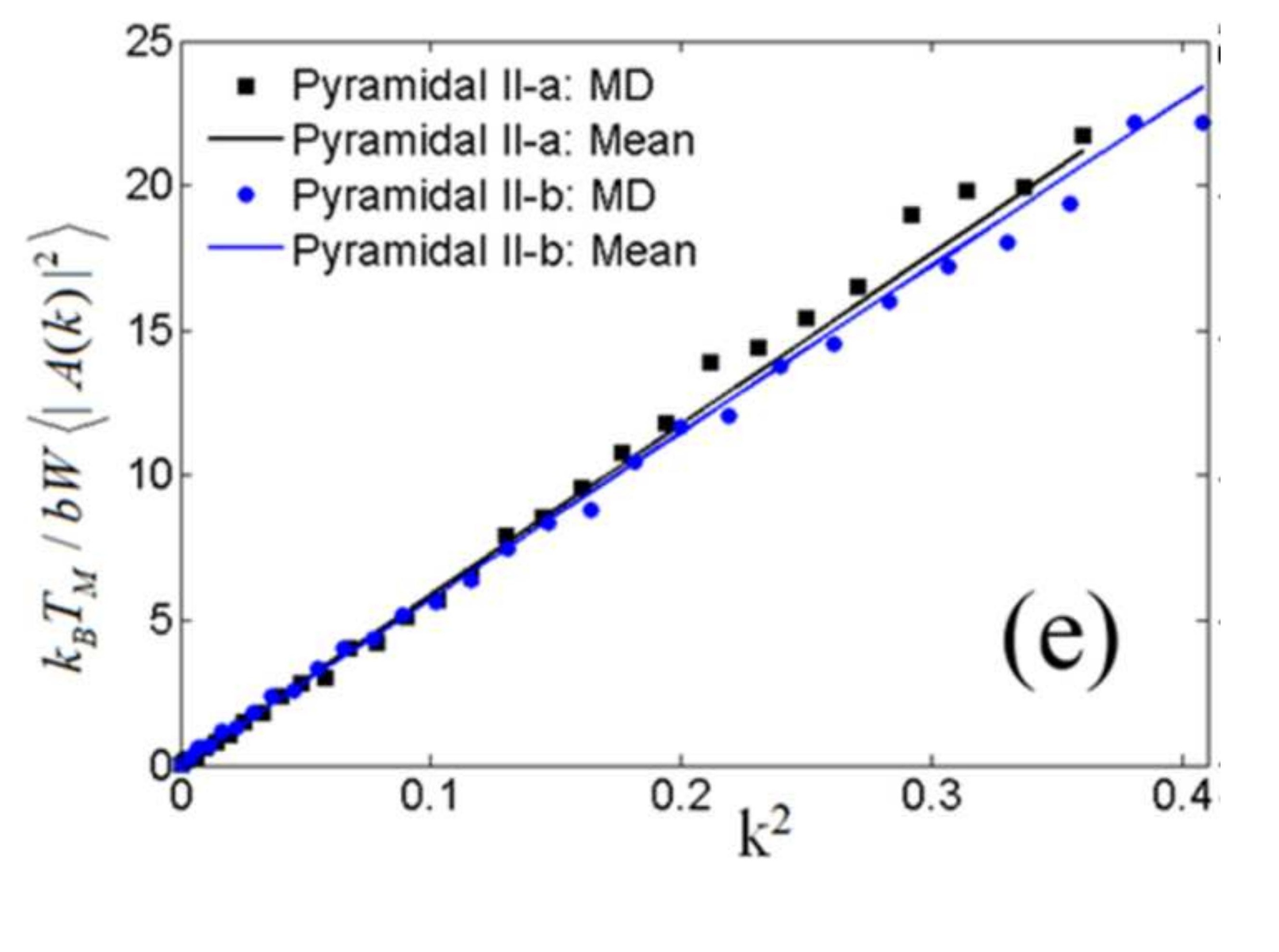

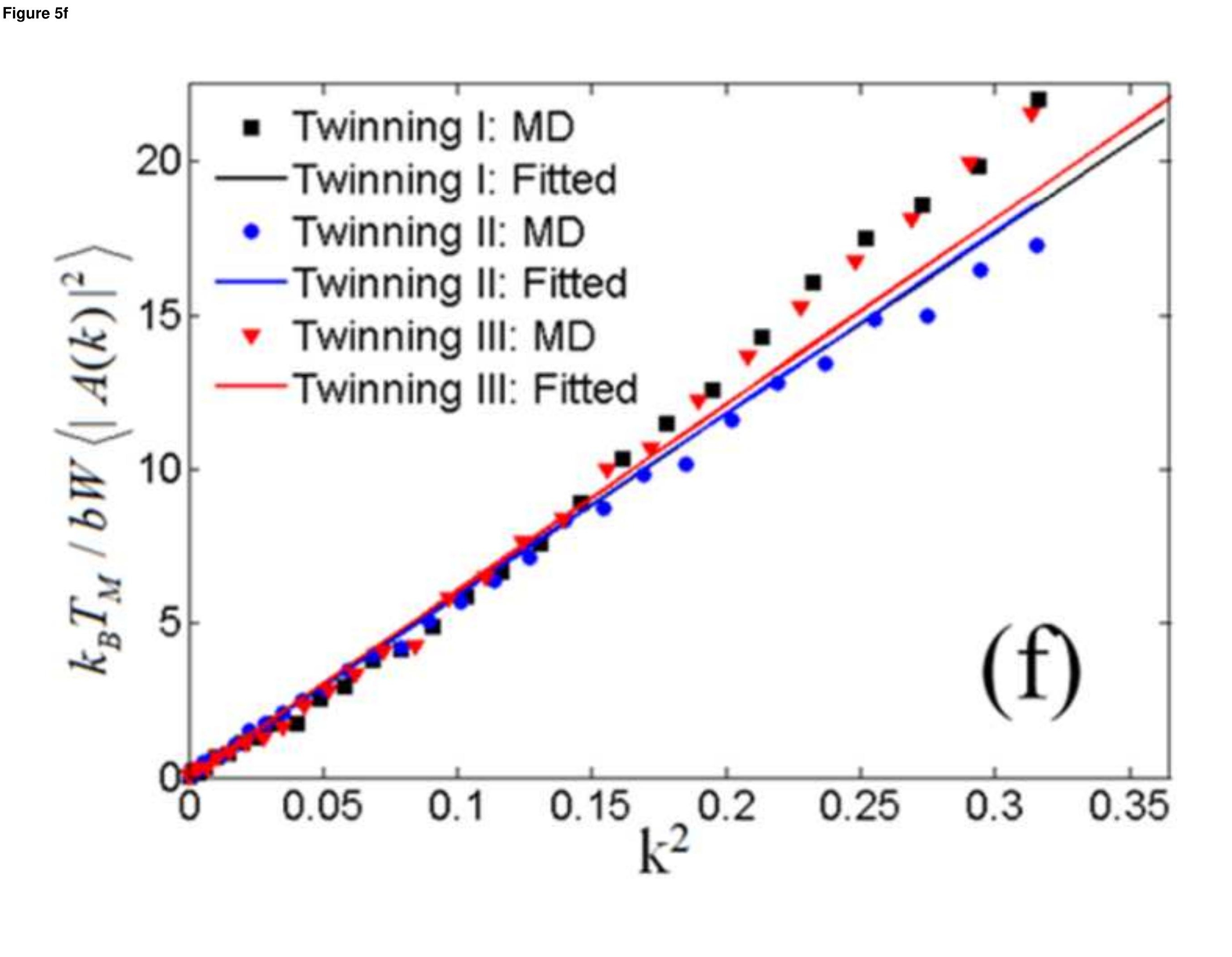


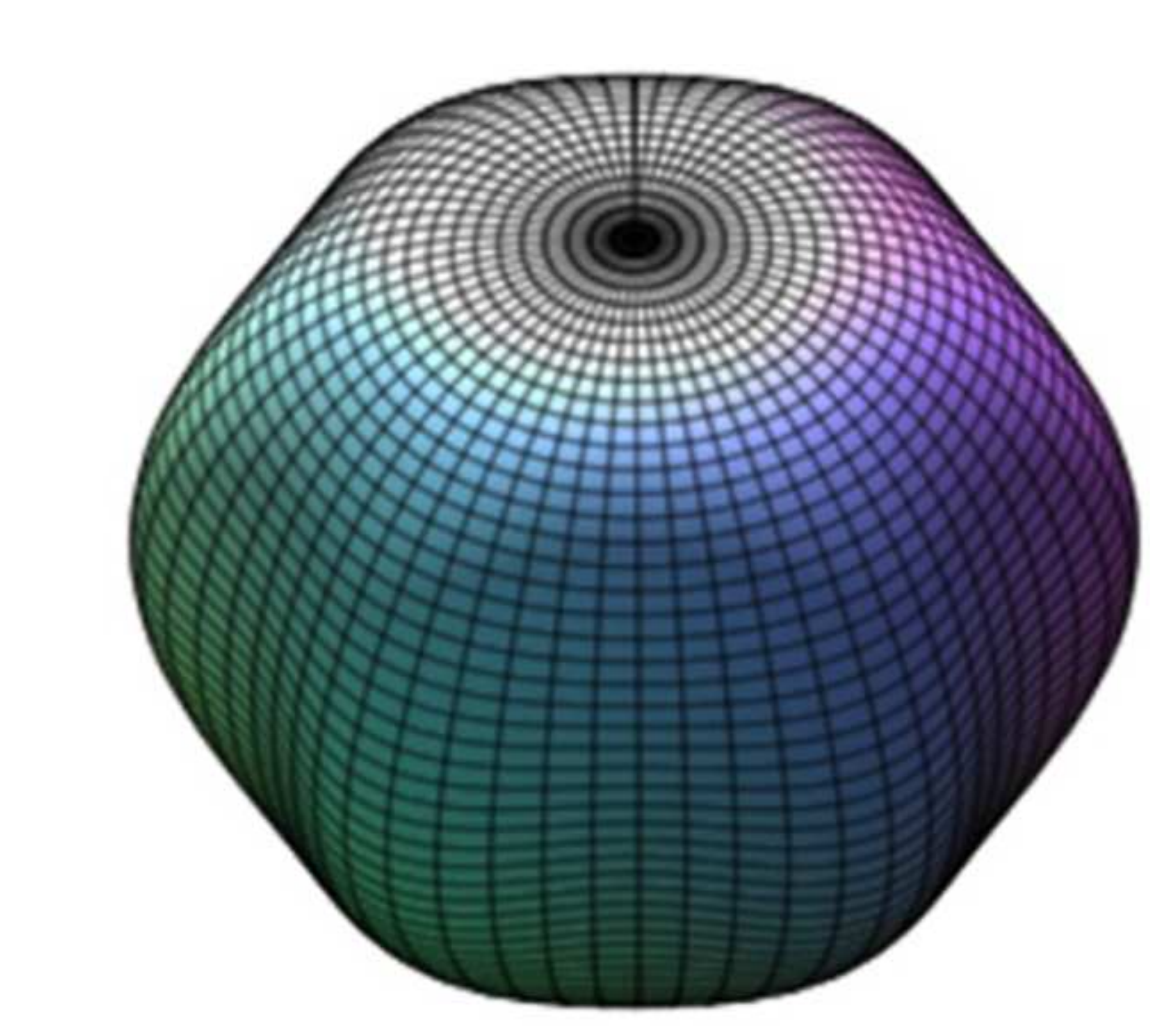

Figure $\epsilon$
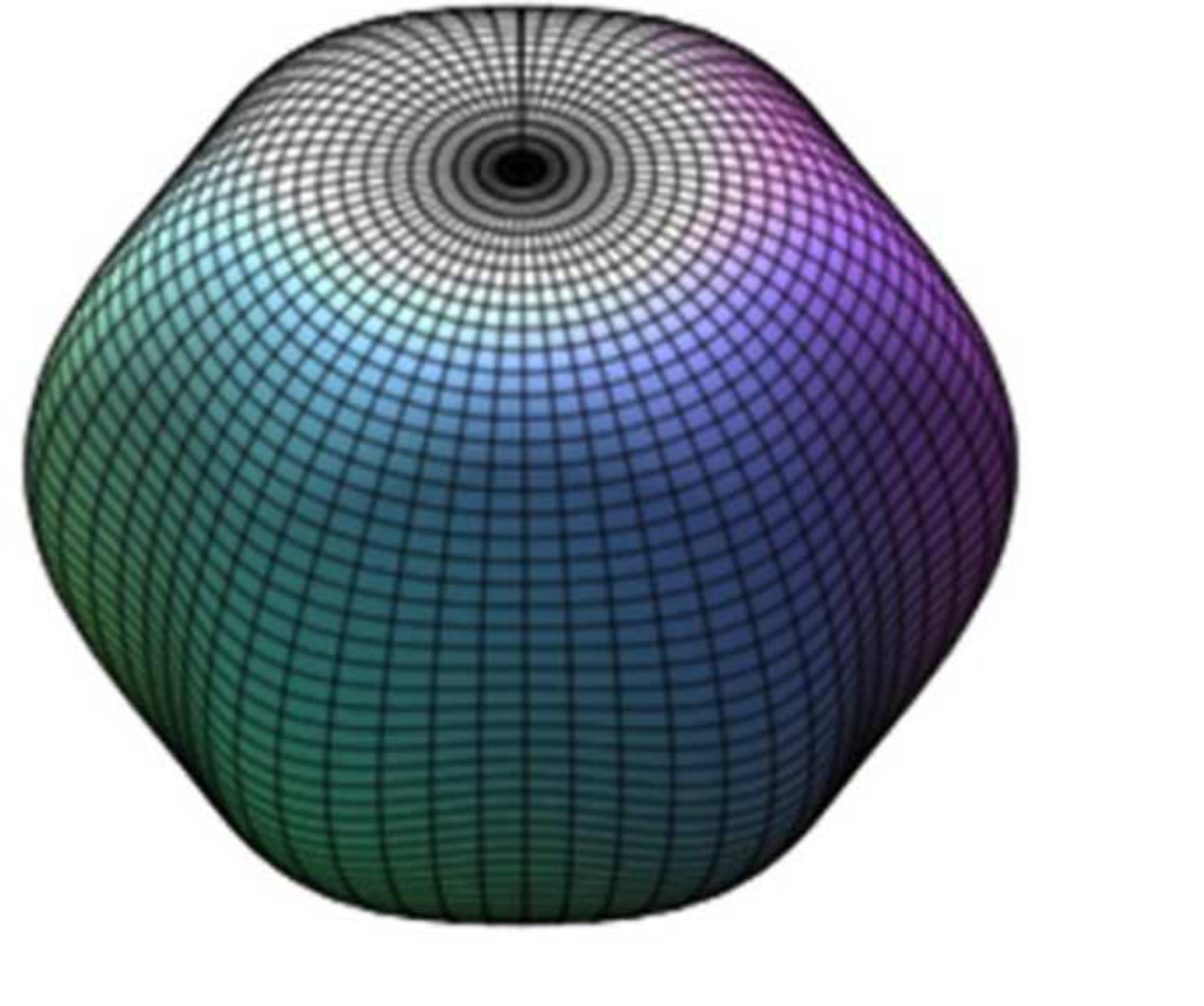


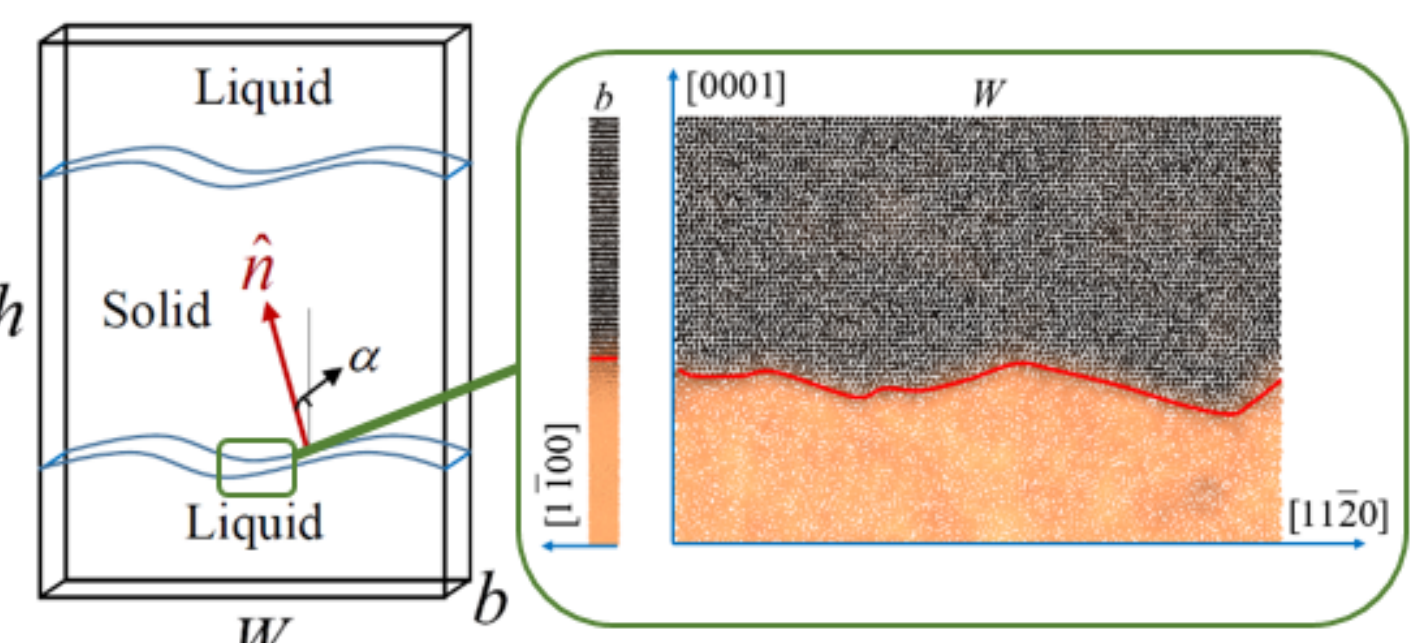

$W$

graphical abstract
- Continuous Order Parameter to Identify Solid-Liquid Interface

- CFM and HCP-symmetry adopted spherical harmonics

- Calculation of HCP-liquid interface stiffness, free energy, and anisotropy

Predicting Preferred Dendrite Growth Direction in Solidification 